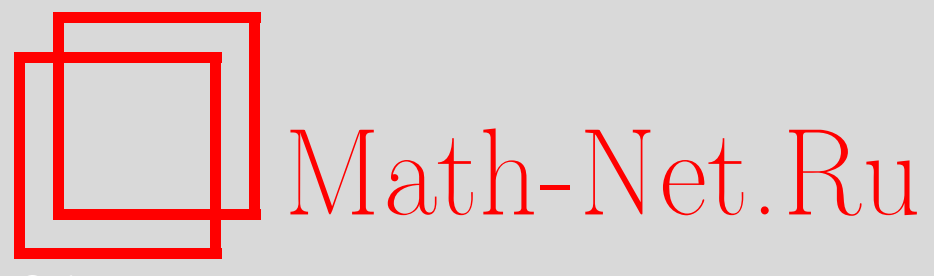

В. В. Чепыжов, О равномерных аттракторах динамических процессов и неавтономных уравнений математической физики, УМH, 2013, том 68, выпуск 2, 159-196

DOI: https://doi.org/10.4213/rm9513

Использование Общероссийского математического портала Math-Net.Ru подразумевает, что вы прочитали и согласны с пользовательским соглашением http: //www. mathnet.ru/rus/agreement

Параметры загрузки:

IP : 52.87 .193 .239

26 апреля 2023 г., 09:41:38

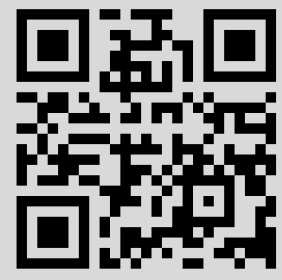




\section{О равномерных аттракторах динамических процессов и неавтономных уравнений математической физики}

\section{В. В. Чепыжов}

Изучаются равномерные аттракторы динамических систем, которые отвечают неавтономным диссипативным уравнениям с частными производными. Задача сводится к исследованию семейств динамических процессов, если исходное уравнение задано на всей оси времени, или к изучению семейств динамических полупроцессов, если уравнение задано на полуоси. Доказаны теоремы о существовании равномерных глобальных аттракторов для семейств процессов и полупроцессов. Изучена структура аттракторов для неавтономных уравнений с трансляционно компактными символами. Найдены условия, при которых аттракторы полупроцессов сводятся к аттракторам соответствующих процессов. Исследован важный частный случай уравнений с асимптотически почти периодическими членами. Рассмотрен ряд примеров неавтономных уравнений математической физики, для которых построены равномерные глобальные аттракторы и изучена их структура.

Библиография: 28 названий.

Ключевые слова: динамические процессы и полупроцессы, трансляционно-компактные функции, равномерные глобальные аттракторы, неавтономные уравнения с частными производными.

DOI: $10.4213 / \mathrm{rm} 9513$

\section{СоДЕРЖАНИЕ}

Введение . . ........................................ 160

1. Процессы, полупроцессы и их равномерные аттракторы........... 162

2. Неавтономное уравнение, его символ и соответствующее семейство

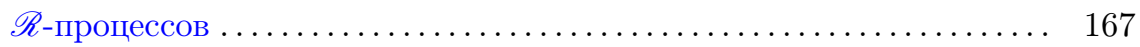

3. Сведение к полугруппе в расширенном фазовом пространстве. Косое произведение ................................. 172

4. О структуре равномерных глобальных аттракторов семейств процессов.

Приложения к УрЧП .............................. 175

5. Неавтономные уравнения с трансляционно компактными символами на $\mathbb{R}_{+}$и их равномерные аттракторы................ 183

Работа выполнена при поддержке РФФИ (гранты № 11-01-00339, 12-01-00203). 
6. Продолжение полупроцессов до процессов . . . . . . . . . . . . . . . .

7. Неавтономные уравнения с асимптотически почти периодическими сим-

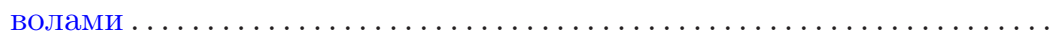

8. Каскадные системы и их глобальные аттракторы .................. 191

Список литературы ..................................... 194

\section{Введение}

Эта работа посвящается светлой памяти Марка Иосифовича Вишика, моего учителя, соавтора и друга. Наше сотрудничество продолжалось без малого 30 лет, начиная с 80-х годов. Одним из результатов его стал цикл работ по теории аттракторов неавтономных уравнений. Многие понятия и термины, которые используются в этой статье, были придуманы Марком Иосифовичем, например, временно́й символ неавтономного уравнения, трансляционно компактная функция, ядро процесса. План этой работы мы обсуждали в мае 2012 г. накануне международной конференции в Москве в честь его 90-летия. Он чувствовал себя неважно, но его, как всегда, переполнял научный энтузиазм, который всегда вдохновлял его учеников и коллег. Тогда я и не думал, что писать эту статью мне придется уже одному. Но надеюсь, что мне передалась частица энтузиазма Марка Иосифовича, которая будет сглаживать боль утраты и помогать в работе уже без моего любимого учителя и друга.

Аттракторы неавтономных динамических процессов, отвечающих различным диссипативным неавтономным уравнениям математической физики, привлекают пристальное внимание исследователей уже более 20 лет. Эта сфера исследований возникла как обобщение теории глобальных аттракторов динамических полугрупп, которые описывают финальное поведение решений автономных уравнений (см. [1]-[5] и обширную цитируемую там литературу).

Известно несколько подходов к построению неавтономных динамических систем, отвечающих неавтономным уравнениям (см. монографии [6]-[8]). Чаще других используется следующий метод, первоначально предложенный в работе [6]. Рассматривается неавтономное эволюционное уравнение на всей оси времени следующего вида:

$$
\partial_{t} u=A_{\sigma(t)}(u), \quad t \geqslant \tau,\left.\quad u\right|_{t=\tau}=u_{\tau} \in E \quad(\tau \in \mathbb{R}) .
$$

Здесь $\sigma(t)$ является функциональным параметром, $\sigma(\cdot): \mathbb{R} \rightarrow \mathscr{M}$, где $\mathscr{M}-$ полное метрическое или банахово пространство. Функция $\sigma(t)$ называется временнъим символом уравнения (0.1), который в приложениях состоит из всех членов и коэффициентов уравнения, явно зависящих от времени. Например, в неавтономной 2D-системе Навье-Стокса временны́м символом служит внешняя сила $g(x, t)$. Этот и другие примеры рассмотрены в разделе 4 . В задаче (0.1) пространство начальных данных $E$ обычно является бесконечномерным пространством Банаха. Предполагается, что при каждом $\tau \in \mathbb{R}$ задача Коши (0.1) имеет единственное решение $u(t) \in E, t \geqslant \tau$, которое представимо в виде $u(t)=U_{\sigma}(t, \tau) u_{\tau}, t \geqslant \tau, \tau \in \mathbb{R}$. Семейство операторов $\{U(t, \tau)\}$, действующих в фазовом пространстве $E$, называется динамическим процессом, отвечающим задаче (0.1). 
Для уравнения (0.1) и для соответствующего процесса $\{U(t, \tau)\}$ требуется построить равномерный глобальный аттрактор. Для этого вместе с уравнением (0.1) необходимо рассмотреть семейство уравнений, символы $\sigma(t)$ которых принадлежат оболочке $\mathscr{H}\left(\sigma_{0}\right):=\left[\sigma_{0}(t+h) \mid h \in \mathbb{R}\right]_{\Xi}$ некоторого исходного символа $\sigma_{0}(t)$. Здесь $[\cdot]_{\Xi}$ обозначает замыкание в топологическом пространстве $\Xi$, которое подбирается в зависимости от свойств изучаемого уравнения. Функция $\sigma(t)$ называется трансляционно компактной в $\Xi$, если ее оболочка $\mathscr{H}\left(\sigma_{0}\right)$ компактна в $\Xi$. Например, если $\Xi=C_{\mathrm{b}}(\mathbb{R} ; \mathscr{M})$ - пространство ограниченных непрерывных функций с топологией равномерной сходимости на всей оси времени, то, согласно критерию Бохнера-Америо, свойство трансляционной компактности эквивалентно почти периодичности. Другие примеры трансляционно компактных функций рассмотрены в разделе 2. Предполагается, что для уравнения (0.1) исходный символ $\sigma_{0}$ является трансляционно компактной функцией в некотором пространстве $\Xi$ и для каждого символа $\sigma \in \mathscr{H}\left(\sigma_{0}\right)$ уравнению (0.1) отвечает процесс $\left\{U_{\sigma}(t, \tau)\right\}$.

Равномерным глобальным аттрактором семейства процессов $\left\{U_{\sigma}(t, \tau)\right\}, \sigma \in$ $\mathscr{H}\left(\sigma_{0}\right)$, называется компактное множество $\mathscr{A}_{\mathscr{H}\left(\sigma_{0}\right)} \Subset E$, которое равномерно $\left(\right.$ по $\left.\sigma \in \mathscr{H}\left(\sigma_{0}\right)\right)$ притягивает любое ограниченное множество начальных данных $B \subset E$ :

$$
\sup _{\sigma \in \Sigma} \operatorname{dist}_{E}\left(U_{\sigma}(t, \tau) B, \mathscr{A}_{\mathscr{H}\left(\sigma_{0}\right)}\right) \rightarrow 0 \quad(t \rightarrow+\infty),
$$

и при этом $\mathscr{A}_{\mathscr{H}\left(\sigma_{0}\right)}$ является минимальным компактным равномерно притягивающим множеством. Здесь $\operatorname{dist}_{E}(\cdot, \cdot)$ обозначает отклонение множеств по Хаусдорфу в метрике пространства $E$ (см. подробнее в разделе 1$)$.

В работе формулируются условия, при которых общие семейства процессов имеют равномерные аттракторы. Отметим, что при построении равномерного аттрактора используется метод косого произведения, т. е. рассматривается динамическая полугруппа $\{\mathscr{S}(h), h \geqslant 0\}$, отвечающая изучаемому семейству процессов $\left\{U_{\sigma}(t, \tau)\right\}, \sigma \in \mathscr{H}\left(\sigma_{0}\right)$, и действующая в расширенном фазовом пространстве $E \times \mathscr{H}\left(\sigma_{0}\right)$ по формуле $\mathscr{S}(h)(u, \sigma)=\left(U_{\sigma}(h, 0) u, \sigma(t+h)\right), h \geqslant 0$, при каждом $(u, \sigma(t)) \in E \times \mathscr{H}\left(\sigma_{0}\right)$. При определенных условиях равномерной диссипативности полугруппа $\{\mathscr{S}(h)\}$ имеет глобальный аттрактор $\mathbf{A}$, причем проекция этого множества на компоненту $E$ расширенного фазового пространства, $\Pi_{1} \mathbf{A}=: \mathscr{A}_{\mathscr{H}\left(\sigma_{0}\right)}$, служит равномерным глобальным аттрактором семейства процессов $\left\{U_{\sigma}(t, \tau)\right\}, \sigma \in \mathscr{H}\left(\sigma_{0}\right)$ (см. раздел 3$)$. Отметим, что из свойства строгой инвариантности получается следующая удобная формула для представления равномерного аттрактора:

$$
\mathscr{A}_{\mathscr{H}\left(\sigma_{0}\right)}:=\bigcup_{\sigma \in \mathscr{H}\left(\sigma_{0}\right)} \mathscr{K}_{\sigma}(0) .
$$

Здесь $\mathscr{K}_{\sigma}(0) \subset E$ обозначает сечение ядра $\mathscr{K}_{\sigma}$ процесса $\left\{U_{\sigma}(t, \tau)\right\}$ в момент времени $t=0$, а ядро процесса - это объединение всех его ограниченных полных траекторий (см. раздел 4).

Описанный выше метод используется в разделе 4 для построения глобальных аттракторов ряда неавтономных нелинейных уравнений математической физики: 2D-системы Навье-Стокса, системы реакции-диффузии и волнового уравнения с диссипацией. 
Следует, однако, отметить, что большой класс неавтономных эволюционных уравнений обладает тем свойством, что его неавтономные члены, зависящие от времени, которые формируют символ $\sigma(t)$ задачи, определены только при $t \geqslant 0$, а их эволюция при $t \leqslant 0$ неизвестна. Такая постановка вполне физически оправдана. Следовательно, соответствующее уравнение (0.1) задано лишь на полуоси времени $\mathbb{R}_{+}:=\{t \geqslant 0\}$.

Это наблюдение приводит ко второму методу построения неавтономной динамической системы для уравнения (0.1) при $t \in \mathbb{R}_{+}$, которая задана на полуоси времени. Отличие от изложенной выше схемы для уравнения $(0.1)$ при $t \in \mathbb{R}$ заключается лишь в том, что все объекты определены и действуют на полуоси $\mathbb{R}_{+}$, а не на всей оси времени. Например, временно́й символ есть функция $\sigma(t), t \geqslant 0$. Оболочка символа $\sigma_{0}(t)$ есть множество

$$
\mathscr{H}\left(\sigma_{0}\right)=\left[\sigma_{0}(t+h) \mid h \geqslant 0\right]_{\Xi} .
$$

Вместо процессов получаются динамические полупрощессъ $\left\{U_{\sigma}(t, \tau) \mid t \geqslant \tau, \tau \geqslant\right.$ $0\}$. Равномерный глобальный аттрактор семейства полупроцессов $\left\{U_{\sigma}(t, \tau)\right\}$, $\sigma \in \mathscr{H}\left(\sigma_{0}\right)$, определяется аналогично (0.2). Наконец, для семейства полупроцессов можно построить косое произведение $\{\mathscr{S}(t), t \geqslant 0\}$ в соответствующем расширенном фазовом пространстве и доказать теорему о существовании равномерного глобального аттрактора $\mathscr{A}_{\mathscr{H}\left(\sigma_{0}\right)}($ см. раздел 5$)$.

Однако далее начинается отличие от процессов. Формулу (0.3) нельзя получить для семейств полупроцессов, так как у полупроцесса нет полных траекторий и, соответственно, для него нельзя определить понятие ядра. Тем не менее при определенных условиях можно описать структуру равномерного глобального аттрактора семейства полупроцессов $\left\{U_{\sigma}(t, \tau)\right\}, \sigma \in \mathscr{H}\left(\sigma_{0}\right)$, с помощью ядер подходящего семейства процессов $\left\{\widetilde{U}_{\sigma}(t, \tau)\right\}, \sigma \in \mathscr{H}\left(\widetilde{\sigma}_{0}\right)$, у которого $\mathscr{H}\left(\widetilde{\sigma}_{0}\right) \subset \mathscr{H}\left(\sigma_{0}\right)$, причем $\widetilde{U}_{\sigma}(t, \tau)=U_{\sigma}(t, \tau)$ при всех $t \geqslant \tau, \tau \geqslant 0$ и при $\sigma \in \mathscr{H}\left(\widetilde{\sigma}_{0}\right)$. Для семейства процессов $\left\{\widetilde{U}_{\sigma}(t, \tau)\right\}, \sigma \in \mathscr{H}\left(\widetilde{\sigma}_{0}\right)$, строится равномерный глобальный аттрактор $\mathscr{A}_{\mathscr{H}}\left(\widetilde{\sigma}_{0}\right)$, для него справедлива формула (0.3), в которой следует заменить $\sigma_{0}$ на $\widetilde{\sigma}_{0}$, и, наконец,

$$
\mathscr{A}_{\mathscr{H}\left(\sigma_{0}\right)}=\mathscr{A}_{\mathscr{H}\left(\widetilde{\sigma}_{0}\right)}=\bigcup_{\sigma \in \mathscr{H}\left(\widetilde{\sigma}_{0}\right)} \mathscr{K}_{\sigma}(0) .
$$

Отметим, что такое семейство процессов единственно (см. подробно в разделе 6$)$.

В разделах 7, 8 рассмотрены два класса уравнений вида (0.1), у которых символ задан на полуоси времени: уравнения с асимптотически почти периодическими символами и так называемые каскадные системы. Для этих уравнений можно построить равномерные глобальные аттракторы соответствующих семейств полупроцессов и свести изучение их структуры к исследованию подходящего семейства процессов.

\section{1. Процессы, полупроцессы и их равномерные аттракторы}

Через $\mathscr{R}$ будет обозначаться одно из двух множеств - временна́я ось $\mathbb{R}$ или полуось $\mathbb{R}_{+}$:

$$
\mathscr{R}=\mathbb{R}:=\{-\infty<t<\infty\} \text { или } \mathscr{R}=\mathbb{R}_{+}:=\{0 \leqslant t<+\infty\} .
$$

Там, где это потребуется, вместо $\mathscr{R}$ будет использоваться $\mathbb{R}$ или $\mathbb{R}_{+}$. 
Неавтономные динамические системы принято описывать с помощью процессов или полупроцессов, действующих в соответствующих фазовых пространствах.

Пусть $E$ - полное метрическое или банахово пространство, на котором задано двухпараметрическое семейство отображений $\{U(t, \tau) \mid t \geqslant \tau, \tau \in \mathscr{R}\}$, $U(t, \tau): E \rightarrow E$. Предполагается, что эти отображения удовлетворяют следующим соотношениям:

$$
\begin{aligned}
U(t, s) \circ U(s, \tau) & =U(t, \tau) \quad \forall t \geqslant s \geqslant \tau, \quad \tau \in \mathscr{R}, \\
U(\tau, \tau) & =\mathrm{Id} \quad \forall \tau \in \mathscr{R} .
\end{aligned}
$$

Здесь Id обозначает тождественный оператор.

ОПРЕДЕЛЕНиЕ 1.1. При $\mathscr{R}=\mathbb{R}$ семейство отображений $\{U(t, \tau)\}:=\{U(t, \tau) \mid$ $t \geqslant \tau, \tau \in \mathbb{R}\}$, которое удовлетворяет (1.2)-(1.3), называется динамическим проиессом в $E$.

При $\mathscr{R}=\mathbb{R}_{+}$такое семейство отображений $\{U(t, \tau)\}:=\{U(t, \tau) \mid t \geqslant \tau$, $\left.\tau \in \mathbb{R}_{+}\right\}$называется динамическим полупрочессом в $E$.

В этом разделе будет сформулирован ряд определений и свойств, относящихся как к процессам, так и к полупроцессам, поэтому для краткости оба этих семейства здесь называются $\mathscr{R}$-процессами. Там, где это важно, будет уточняться, к какому из двух семейств отображений относится то или иное свойство.

Через $\mathscr{B}(E)$ обозначается совокупность всех ограниченных подмножеств в $E$.

Динамический $\mathscr{R}$-процесс $\{U(t, \tau)\}$ называется ограниченным, если для любого $B \in \mathscr{B}(E)$ найдется $B_{1} \in \mathscr{B}(E)$ такое, что $U(t, \tau) B \subset B_{1}$ при всех $t \geqslant \tau$, $\tau \in \mathscr{R}$.

Следующие два понятия описывают свойства диссипативности неавтономных динамических систем. Множество $B_{0} \subset E$ называется поглощающим для $\mathscr{R}$-процесса $\{U(t, \tau)\}$, если для любых $\tau \in \mathscr{R}$ и $B \in \mathscr{B}(E)$ найдется $t_{0}=$ $t_{0}(\tau, B) \geqslant \tau$ такое, что

$$
U(t, \tau) B \subseteq B_{0} \quad \text { при всех } t \geqslant t_{0} .
$$

Множество $P \subset E$ называется притягивающим для $\mathscr{R}$-процесса $\{U(t, \tau)\}$, если для каждого $\tau \in \mathscr{R}$ и любого $B \in \mathscr{B}(E)$

$$
\operatorname{dist}_{E}(U(t, \tau) B, P) \rightarrow 0 \quad(t \rightarrow+\infty),
$$

где $\operatorname{dist}_{E}(X, Y)$ обозначает стандартное отклонение по Хаусдорфу в метрике $E$ подмножества $X$ от подмножества $Y$ (см., например, [1]). Свойство (1.4) можно сформулировать в следующем эквивалентном виде: для любого $\varepsilon>0$ множество $\mathscr{O}_{\varepsilon}(P)$ является поглощающим для $\mathscr{R}$-процесса $\{U(t, \tau)\}$ (здесь $\mathscr{O}_{\varepsilon}(X)$ обозначает $\varepsilon$-окрестность подмножества $X$ в пространстве $E)$, т. е. для любых $\tau \in \mathscr{R}$ и $B \in \mathscr{B}(E)$ найдется $t_{0}=t_{0}(\varepsilon, \tau, B) \geqslant \tau$ такое, что

$$
U(t, \tau) B \subseteq \mathscr{O}_{\varepsilon}(P) \quad \text { при всех } t \geqslant t_{0} .
$$


Компактным называется $\mathscr{R}$-процесс, у которого имеется компактное в $E$ поглощающее множество. Асимптотически компактным называется $\mathscr{R}$-процесс, у которого есть компактное притягивающее множество.

Будут рассматриваться семейства $\mathscr{R}$-процессов $\left\{U_{\sigma}(t, \tau)\right\}, \sigma \in \Sigma$, которые зависят от некоторого функционального параметра $\sigma$. Параметр $\sigma$ называется символом $\mathscr{R}$-процесса $\left\{U_{\sigma}(t, \tau)\right\}$, а множество $\Sigma$ - пространством символов.

Введенные выше понятия, связанные с фиксированным $\mathscr{R}$-процессом $\{U(t, \tau)\}$, легко обобщаются на семейства $\mathscr{R}$-процессов $\left\{U_{\sigma}(t, \tau)\right\}, \sigma \in \Sigma$.

ОПРедЕЛЕНИЕ 1.2. Семейство $\mathscr{R}$-процессов $\left\{U_{\sigma}(t, \tau)\right\}, \sigma \in \Sigma$, называется равномерно (по $\sigma \in \Sigma$ ) ограниченным, если для любого $B \in \mathscr{B}(E)$ найдется $B_{1} \in \mathscr{B}(E)$ такое, что $U_{\sigma}(t, \tau) B \subset B_{1}$ при всех $t \geqslant \tau, \tau \in \mathscr{R}, \sigma \in \Sigma$.

ОПРЕДЕЛЕНИЕ 1.3. Множество $B_{0} \subset E$ называется равномерно $($ по $\sigma \in \Sigma$ ) поглощающим для семейства $\mathscr{R}$-процессов $\left\{U_{\sigma}(t, \tau)\right\}, \sigma \in \Sigma$, если для любых $\tau \in \mathscr{R}$ и $B \in \mathscr{B}(E)$ найдется $t_{0}=t_{0}(\tau, B) \geqslant \tau$ такое, что

$$
\bigcup_{\sigma \in \Sigma} U_{\sigma}(t, \tau) B \subseteq B_{0} \quad \text { при всех } t \geqslant t_{0} .
$$

ОПРеДЕЛЕНИЕ 1.4. Множество $P \subset E$ называется равномерно $($ по $\sigma \in \Sigma$ ) притягивающим для семейства $\mathscr{R}$-процессов $\left\{U_{\sigma}(t, \tau)\right\}, \sigma \in \Sigma$, если для каждого $\tau \in \mathscr{R}$ и любого $B \in \mathscr{B}(E)$

$$
\sup _{\sigma \in \Sigma} \operatorname{dist}_{E}\left(U_{\sigma}(t, \tau) B, P\right) \rightarrow 0 \quad(t \rightarrow+\infty),
$$

другими словами, для любого $\varepsilon>0$ множество $\mathscr{O}_{\varepsilon}(P)$ является равномерно поглощающим для $\left\{U_{\sigma}(t, \tau)\right\}, \sigma \in \Sigma$ : для любых $\tau \in \mathscr{R}$ и $B \in \mathscr{B}(E)$ найдется $t_{0}=t_{0}(\varepsilon, \tau, B) \geqslant \tau$ такое, что

$$
\bigcup_{\sigma \in \Sigma} U_{\sigma}(t, \tau) B \subseteq \mathscr{O}_{\varepsilon}(P) \text { при всех } t \geqslant t_{0} .
$$

Семейство $\mathscr{R}$-процессов, у которого имеется компактное (в топологии $E$ ) равномерно поглощающее множество, называется равномерно компактным. Семейство $\mathscr{R}$-процессов, у которого есть компактное равномерно притягивающее множество, называется равномерно асимптотически компактным. Отметим, что равномерно асимптотически компактное семейство $\mathscr{R}$-процессов является одновременно равномерно компактным. Обратное утверждение в общем случае не верно.

Сформулируем основное определение равномерного глобального аттрактора семейства $\mathscr{R}$-процессов.

ОПРеДЕЛЕНИЕ 1.5. Замкнутое множество $\mathscr{A}_{\Sigma} \subset E$ называется равномерным $($ по $\sigma \in \Sigma$ ) глобальным аттрактором семейства динамических $\mathscr{R}$-процессов $\left\{U_{\sigma}(t, \tau)\right\}, \sigma \in \Sigma$, если оно является равномерно притягивающим для этого семейства (свойство притяжения) и содержится в любом замкнутом равномерно притягивающем множестве этого семейства $\mathscr{R}$-процессов (свойство минимальности). 
Отметим, что в силу свойства минимальности равномерный глобальный аттрактор определяется однозначным образом. Это понятие было введено в работе [6] (см. также [7], [9]--[11]).

Для произвольного множества $B \in \mathscr{B}(E)$ строится равномерное $\omega$-предельное множество $\omega_{\tau, \Sigma}(B)$ с истоком $\tau \in \mathscr{R}$, которое зависит от пространства символов $\Sigma$ :

$$
\omega_{\tau, \Sigma}(B)=\bigcap_{t \geqslant \tau}\left[\bigcup_{\sigma \in \Sigma} \bigcup_{s \geqslant t} U_{\sigma}(s, \tau) B\right]_{E} .
$$

Здесь и далее квадратные скобки $[\cdot]_{E}$ обозначают замыкание в пространстве $E$.

УтВеРЖДЕНИЕ 1.1. Если семейство $\mathscr{R}$-процессов $\left\{U_{\sigma}(t, \tau)\right\}, \sigma \in \Sigma$, имеет компактное равномерно притягивающее множество $P$, то для любого $\tau \in \mathscr{R}$ и любого $B \in \mathscr{B}(E)$

(i) множество $\omega_{\tau, \Sigma}(B)$ непусто, компактно в $E$ u $\omega_{\tau, \Sigma}(B) \subseteq P$;

(ii) $\sup _{\sigma \in \Sigma} \operatorname{dist}_{E}\left(U_{\sigma}(t, \tau) B, \omega_{\tau, \Sigma}(B)\right) \rightarrow 0(t \rightarrow+\infty)$;

(iii) если $Y$ замкнуто в $E u \sup _{\sigma \in \Sigma} \operatorname{dist}_{E}\left(U_{\sigma}(t, \tau) B, Y\right) \rightarrow 0(t \rightarrow+\infty)$, mo $\omega_{\tau, \Sigma}(B) \subseteq Y$.

ДокАЗАтЕльство. Заметим, что из определения (1.7) множества $\omega_{\tau, \Sigma}(B)$ следует, что

$$
y \in \omega_{\tau, \Sigma}(B) \Leftrightarrow\left\{\begin{array}{l}
\text { существуют }\left\{x_{n}\right\} \subseteq B,\left\{\sigma_{n}\right\} \subseteq \Sigma \text { и }\left\{t_{n}\right\} \subset \mathscr{R} \\
\text { такие, что } t_{n} \rightarrow+\infty \text { и } U_{\sigma}\left(t_{n}, \tau\right) x_{n} \rightarrow y .
\end{array}\right.
$$

(i) Покажем, что $\omega_{\tau, \Sigma}(B) \neq \varnothing$. Для любых фиксированных $\sigma \in \Sigma$ и $x \in B$ рассмотрим произвольную последовательность $t_{n} \rightarrow+\infty$. В силу свойства (1.6) равномерного притяжения к $P$ мы имеем $\operatorname{dist}_{E}\left(U_{\sigma}\left(t_{n}, \tau\right) x, P\right) \rightarrow 0$, значит, $\left\|U_{\sigma}\left(t_{n}, \tau\right) x-y_{n}\right\|_{E} \rightarrow 0$ для некоторой последовательности $\left\{y_{n}\right\} \subseteq P$.

Множество $P$ компактно, следовательно, из $\left\{y_{n}\right\}$ можно выделить подпоследовательность $\left\{y_{n^{\prime}}\right\}$, которая сходится к некоторой точке $y \in P$. Тогда, очевидно, $U_{\sigma}\left(t_{n^{\prime}}, \tau\right) x \rightarrow y$. Учитывая (1.8), получаем, что построенная точка $y$ принадлежит $\omega_{\tau, \Sigma}(B)$, т. е. $\omega_{\tau, \Sigma}(B) \neq \varnothing$.

Проверим, что $\omega_{\tau, \Sigma}(B) \subseteq P$. Пусть $y \in \omega_{\tau, \Sigma}(B)$ и $\left\{x_{n}\right\} \subseteq B,\left\{t_{n}\right\} \subseteq \mathscr{R}$, $\left\{\sigma_{n}\right\} \subset \Sigma-$ последовательности, определенные в (1.8). С помощью свойства равномерного притяжения к $P$ (см. (1.6)) получаем, что

$$
\operatorname{dist}_{E}\left(U_{\sigma}\left(t_{n}, \tau\right) x_{n}, P\right) \rightarrow 0 .
$$

Тогда $\operatorname{dist}_{E}(y, P)=0$. Множество $P$ замкнуто, тем самым, $y \in P$ для любого $y \in \omega_{\tau, \Sigma}(B)$ и $\omega_{\tau, \Sigma}(B) \subseteq P$. Отсюда заключаем, что $\omega_{\tau, \Sigma}(B)$ компактно, поскольку $\omega_{\tau, \Sigma}(B)$ по определению замкнуто (см. (1.7)).

(ii) Предположим обратное: для некоторого $B \in \mathscr{B}(E)$

$$
\sup _{\sigma \in \Sigma} \operatorname{dist}_{E}\left(U_{\sigma}(t, \tau) B, \omega_{\tau, \Sigma}(B)\right) \nrightarrow 0 \quad(t \rightarrow+\infty),
$$

т. е. для некоторых последовательностей $\left\{x_{n}\right\} \subseteq B,\left\{\sigma_{n}\right\} \subseteq \Sigma,\left\{t_{n}\right\} \subset \mathscr{R}\left(t_{n} \rightarrow\right.$ $+\infty)$

$$
\operatorname{dist}_{E}\left(U_{\sigma_{n}}\left(t_{n}, \tau\right) x_{n}, \omega_{\tau, \Sigma}(B)\right) \geqslant \delta>0 \quad \forall n \in \mathbb{N} \text {. }
$$


Свойство равномерного притяжения к $P$ дает $\operatorname{dist}_{E}\left(U_{\sigma_{n}}\left(t_{n}, \tau\right) x_{n}, P\right) \rightarrow 0$. Опять находим последовательность $\left\{y_{n}\right\} \subset P$ такую, что

$$
\left\|U_{\sigma_{n}}\left(t_{n}, \tau\right) x_{n}-y_{n}\right\|_{E} \rightarrow 0
$$

Множество $P$ компактно, поэтому можем предполагать, что $y_{n} \rightarrow y, y \in P$, т. е. $U_{\sigma_{n}}\left(t_{n}, \tau\right) x_{n} \rightarrow y$, и в силу (1.8) $y \in \omega_{\tau, \Sigma}(B)$. Однако из (1.9) следует, что $\operatorname{dist}_{E}\left(y, \omega_{\tau, \Sigma}(B)\right) \geqslant \delta>0$. Пришли к противоречию.

(iii) Пусть $Y$ - замкнутое равномерно (по $\sigma \in \Sigma$ ) притягивающее множество семейства $\mathscr{R}$-процессов $\left\{U_{\sigma}(t, \tau)\right\}, \sigma \in \Sigma$. Если $y \in \omega_{\tau, \Sigma}(B)$, то ввиду (1.8) для некоторых $\left\{x_{n}\right\} \subseteq B,\left\{\sigma_{n}\right\} \subseteq \Sigma,\left\{t_{n}\right\} \subset \mathscr{R}$ заключаем, что

$$
U_{\sigma_{n}}\left(t_{n}, \tau\right) x_{n} \rightarrow y \quad\left(t_{n} \rightarrow+\infty\right)
$$

Поскольку множество $Y$ равномерно притягивает, то $\operatorname{dist}_{E}\left(U_{\sigma_{n}}\left(t_{n}, \tau\right) x_{n}, Y\right) \rightarrow 0$, и, следовательно, $\operatorname{dist}_{E}(y, Y)=0$, т. е. $y \in Y$. Поэтому $\omega_{\tau, \Sigma}(B) \subseteq Y$. Утверждение полностью доказано.

С помощью утверждения 1.1 устанавливается следующая общая теорема.

Теорема 1.1. Если семейство $\mathscr{R}$-процессов $\left\{U_{\sigma}(t, \tau)\right\}, \sigma \in \Sigma$, является равномерно (по $\sigma \in \Sigma$ ) асимптотически компактным, то оно имеет компактный (в E) равномерный глобальный аттрактор $\mathscr{A}_{\Sigma}$.

ДокАЗАТЕЛЬство. Покажем, что множество

$$
\mathscr{A}_{\Sigma}=\left[\bigcup_{\tau \in \mathscr{R}} \bigcup_{n \in \mathbb{N}} \omega_{\tau, \Sigma}\left(B_{n}\right)\right]_{E}
$$

служит искомым равномерным аттрактором. Здесь $B_{n}=\left\{x \in E \mid\|x\|_{E} \leqslant n\right\}-$ шар в $E$ с радиусом $n \in \mathbb{N}$. Действительно, из утверждения 1.1 , (i) следует, что $\mathscr{A}_{\Sigma} \subseteq P$. Кроме того, если $B \subseteq \mathscr{B}(E)$, то $B \subseteq B_{n}$ для некоторого $n \in \mathbb{N}$, и, следовательно, $\omega_{\tau, \Sigma}(B) \subseteq \omega_{\tau, \Sigma}\left(B_{n}\right) \subseteq \mathscr{A}_{\Sigma}$, т. е. $\mathscr{A}_{\Sigma}$ притягивает $U_{\sigma}(t, \tau) B$ равномерно (по $\sigma \in \Sigma$ ) (см. утверждение 1.1 , (ii)). Одновременно с этим из пункта (iii) утверждения 1.1 следует, что каждое множество $\omega_{\tau, \Sigma}\left(B_{n}\right)$ принадлежит каждому замкнутому равномерно притягивающему множеству. Поэтому множество $\mathscr{A}_{\Sigma}$, определенное в (1.10), обладает свойством минимальности.

СлеДСтвиЕ 1.1. Если семейство $\mathscr{R}$-прочессов $\left\{U_{\sigma}(t, \tau)\right\}, \sigma \in \Sigma$, является равномерно (по $\sigma \in \Sigma$ ) асимптотически компактным, то для любого подмножества $\Sigma^{\prime} \subset \Sigma$ у семейства $\mathscr{R}$-процессов $\left\{U_{\sigma}(t, \tau)\right\}, \sigma \in \Sigma^{\prime}$, существует равномерный (по $\left.\Sigma^{\prime}\right)$ глобальный аттрактор $\mathscr{A}_{\Sigma^{\prime}}$, причем $\mathscr{A}_{\Sigma^{\prime}} \subseteq \mathscr{A}_{\Sigma}$ (как показано в [7], включение может быть строгим).

В частности, для каждого $\mathscr{R}$-процесса $\left\{U_{\sigma}(t, \tau)\right\}$ с фиксированным символом $\sigma \in \Sigma$ можно построить (неравномерный) глобальный аттрактор $\mathscr{A}_{\{\sigma\}}$, причем $\mathscr{A}_{\{\sigma\}} \subseteq \mathscr{A}_{\Sigma}$. Тогда очевидно, что $\bigcup_{\sigma \in \Sigma} \mathscr{A}_{\{\sigma\}} \subseteq \mathscr{A}_{\Sigma}$, однако и здесь включение может быть строгим (см. примеры в [6], [7]). 
ЗАмЕчание 1.1. Легко показать, что если семейство $\mathscr{R}$-процессов $\left\{U_{\sigma}(t, \tau)\right\}$, $\sigma \in \Sigma$, имеет компактное равномерно (по $\sigma \in \Sigma$ ) поглощающее множество $P$, то $\mathscr{A}_{\Sigma}=\omega_{0, \Sigma}(P)$. Если же $P$ является лишь равномерно притягивающим, то $\mathscr{A}_{\Sigma}=\omega_{0, \Sigma}\left(\mathscr{O}_{\varepsilon}(P)\right)$ при любом $\varepsilon>0$, и не известно, можно ли утверждать без дополнительных условий, что $\mathscr{A}_{\Sigma}=\omega_{0, \Sigma}(P)$.

\section{2. Неавтономное уравнение, его символ и соответствующее семейство $\mathscr{R}$-процессов}

Покажем, как можно построить $\mathscr{R}$-процесс, соответствующий некоторому неавтономному эволюционному уравнению. (Напомним, что $\mathscr{R}$ обозначает $\mathbb{R}$ или $\mathbb{R}_{+}$.) Рассматривается неавтономное уравнение следующего вида:

$$
\partial_{t} u=A(u, t), \quad t \geqslant \tau \quad(\tau \in \mathscr{R}) .
$$

Здесь $A(u, t)$ - нелинейный оператор $A(\cdot, t): E_{1} \rightarrow E_{0}$ при каждом $t \in \mathscr{R}$, где $E_{1}$ и $E_{0}$ - банаховы пространства такие, что $E_{1} \subseteq E_{0}$. Изучаются решения $u(t)$ этого уравнения, которые заданы при всех $t \geqslant \tau$. При $t=\tau$ ставится начальное условие:

$$
u(\tau)=\left.u\right|_{t=\tau}=u_{\tau}, \quad u_{\tau} \in E,
$$

где $E$ - пространство Банаха, причем $E_{1} \subseteq E \subseteq E_{0}$. Предполагается, что при каждом $\tau \in \mathscr{R}$ и при любом $u_{\tau} \in E$ задача Коши (2.1)-(2.2) имеет единственное решение $u(t)$ такое, что $u(t) \in E$ при всех $t \geqslant \tau$. Смысл фразы "функция $u(t)$ является решением задачи (2.1)-(2.2)" следует пояснять для каждого конкретного уравнения (см. примеры в [1], [7], [12]).

Изучается семейство отображений $\{U(t, \tau)\}, t \geqslant \tau, \tau \in \mathscr{R}$, которое действует в пространстве $E$ по формуле

$$
U(t, \tau) u_{\tau}=u(t), \quad t \geqslant \tau, \quad \tau \in \mathscr{R},
$$

где $u(t)$ - это решение $(2.1)-(2.2)$ с начальным условием $u_{\tau} \in E$. Поскольку по предположению задача Коши (2.1)-(2.2) однозначно разрешима, то семейство $\{U(t, \tau)\}$ удовлетворяет свойствам (1.2)-(1.3). Следовательно, $\{U(t, \tau)\}$ является $\mathscr{R}$-процессом в $E$.

При изучении конкретных неавтономных эволюционных уравнений с частными производными можно заметить, что зависимость от времени этих уравнений выражается явно в виде соответствующих коэффициентов, внешних сил, параметров сред, функций управления и т. п. Будем обозначать семейство всех зависящих от времени коэффициентов неавтономного уравнения через $\sigma(t)$, $t \in \mathscr{R}$. Параметр $\sigma(t)$ называется временнъим символом (или просто символом) уравнения. Свои значения функция $\sigma(t)$ принимает в некотором метрическом пространстве $\mathscr{M}$ или банаховом пространстве $\mathscr{E}$, которое зависит от конкретной задачи. При таком подходе само неавтономное уравнение (2.1) и соответствующую ей задачу Коши удобно записывать в виде

$$
\begin{aligned}
& \partial_{t} u=A_{\sigma(t)}(u), \quad t \geqslant \tau \quad(\tau \in \mathscr{R}), \\
& \left.u\right|_{t=\tau}=u_{\tau} .
\end{aligned}
$$


Например, в неавтономной 2D-системе Навье-Стокса

$$
\partial_{t} u+\nu L u+B(u, u)=g(x, t)
$$

с зависящей от времени внешней силой $g(x, t) \in C_{\mathrm{b}}(\mathbb{R} ; H)$ временнб́м символом служит $\sigma(t):=g(x, t)$. (Этот и другие примеры будут рассмотрены в разделе 4.)

При описании временнь́х символов уравнений переменная времени $t$ будет заменяться на $s$. Предполагается, что символ $\sigma(s)$, как функция времени $s$, принадлежит некоторому объемлющему пространству

$$
\Xi:=\{\xi(s), s \in \mathscr{R} \mid \xi(s) \in \mathscr{M} \text { при почти всех } s \in \mathscr{R}\},
$$

которое снабжено хаусдорфовой топологией (это условие выполнено, если $\Xi$ является метрическим пространством). В рассмотренном выше примере 2D-системы Навье-Стокса $\mathscr{M}=H$, а объемлющим пространством является $\Xi=$ $C_{\mathrm{b}}(\mathbb{R} ; H)$. Напомним, что топология в пространстве $C_{\mathrm{b}}(\mathbb{R} ; H)$ порождается нормой

$$
\|g(\cdot, \cdot)\|_{C_{\mathrm{b}}(\mathbb{R} ; H)}:=\sup _{s \in \mathbb{R}}\|g(\cdot, s)\|_{H} .
$$

Рассмотрим операторы трансляции по времени $\{T(h), h \in \mathscr{R}\}$, которые действуют в пространстве $\Xi$ по формуле $T(h) \xi(s)=\xi(h+s)$. Предполагается, что оператор $T(h)$ отображает $\Xi$ на себя при каждом $h \in \mathscr{R}$. Тогда при $\mathscr{R}=\mathbb{R}$ операторы $\{T(h), h \in \mathbb{R}\}$ образуют группу, а при $\mathscr{R}=\mathbb{R}_{+}-$полугруппу. Для простоты предполагается, что операторы $T(h)$ непрерывны в топологии $\Xi$.

Как уже отмечалось, мы будем рассматривать семейства уравнений (2.4) с различными временны́ми символами $\sigma(s)$, которые принадлежат некоторому пространству символов $\Sigma \subset \Xi$. Предположим, что пространству $\Sigma$ вместе с каждым его символом $\sigma(s)$ принадлежат все сдвиги $T(h) \sigma(s)=\sigma(s+h) \in \Sigma$, $h \in \mathscr{R}$. Тогда при $\mathscr{R}=\mathbb{R}$ группа $\{T(h)\}$ строго инвариантна на $\Sigma$ :

$$
T(h) \Sigma=\Sigma \quad \forall h \in \mathbb{R},
$$

а при $\mathscr{R}=\mathbb{R}_{+}$полугруппа $\{T(h)\}$ нестрого инвариантна на $\Sigma$ :

$$
T(h) \Sigma \subseteq \Sigma \quad \forall h \in \mathbb{R}_{+} .
$$

Отметим, что при изучении семейств процессов будет использоваться свойство (2.6), а семейств полупроцессов - (2.7). В этом заключается существенная разница этих двух семейств отображений, описывающих неавтономные эволюционные уравнения.

Предположим, что для любого символа $\sigma \in \Sigma$ задача Коши (2.4)-(2.5) однозначно разрешима при любом $\tau \in \mathscr{R}$ и каждом $u_{\tau} \in E$. Порождаемый при этом $\mathscr{R}$-процесс обозначим через $\left\{U_{\sigma}(t, \tau)\right\}$. Изучается семейство $\mathscr{R}$-процессов $\left\{U_{\sigma}(t, \tau)\right\}, \sigma \in \Sigma$.

Заметим, что построенное семейство $\mathscr{R}$-процессов удовлетворяет следующему трансляционному тождеству:

$$
U_{T(h) \sigma}(t, \tau) u_{\tau}=U_{\sigma}(t+h, \tau+h) u_{\tau} \quad \forall h \geqslant 0, \quad t \geqslant \tau, \quad \tau \in \mathscr{R} .
$$


Чтобы доказать тождество (2.8), следует заменить символ $\sigma(t)$ уравнения $(2.4)$ на $T(h) \sigma(t)=\sigma(t+h)$, а потом сделать замену переменной $t+h=t_{1}$. Тождество (2.8) означает, что сдвиг символа уравнения на $h$ эквивалентен нахождению решения уравнения с исходным символом $\sigma(t)$ в момент времени $t+h$ с начальным условием $\left.u\right|_{t=\tau+h}=u_{\tau}$. Здесь используется свойство однозначной разрешимости задачи Коши (2.4)-(2.5) с произвольными $\tau \in \mathscr{R}$ и любыми $u_{\tau} \in E$.

Опишем типичное пространство символов, сопоставляемое неавтономному уравнению. Символ исходного уравнения (2.1) (или (2.4)) обозначается через $\sigma_{0}(s), s \in \mathscr{R}$. Вместе с этим уравнением, имеющим символ $\sigma_{0}(s)$, рассматриваются уравнения (2.4) со всевозможными символами $\sigma_{h}(s)=T(h) \sigma_{0}(s)=$ $\sigma_{0}(h+s)$ при $h \in \mathscr{R}$. Кроме того, рассматриваются уравнения, символы $\sigma(s)$ которых являются пределами всевозможных последовательностей вида $\sigma_{h_{n}}(s)=T\left(h_{n}\right) \sigma_{0}(s)$ при $n \rightarrow \infty$ в топологии пространства $\Xi$. В результате получается оболочка $\mathscr{H}\left(\sigma_{0}\right)$ исходного символа $\sigma_{0}(s)$ в пространстве $\Xi$.

ОПРЕДЕлЕНИЕ 2.1. Множество

$$
\mathscr{H}(\sigma):=[\{\sigma(s+h) \mid h \in \mathscr{R}\}]_{\Xi}
$$

называется оболочкой функции $\sigma(s)$ в пространстве $\Xi$, где $[\cdot]_{\Xi}$ обозначает замыкание в пространстве $\Xi$.

Множество $\mathscr{H}\left(\sigma_{0}\right)$ можно использовать в качестве пространства символов уравнения (2.4). Заметим, что свойства (2.6) или (2.7) выполнены для $\Sigma=\mathscr{H}(\sigma)$.

Будут изучаться уравнения вида $(2.1)$ и (2.4), у которых символы $\sigma_{0}(s)$ являются трансляционно компактными функциями в $\Xi$ (см. также [7], [13]-[15]).

ОПРЕДЕЛЕНИЕ 2.2. Функция $\sigma(\cdot) \in \Xi$ называется трансляиионно компактной в $\Xi$, если ее оболочка $\mathscr{H}(\sigma)$ компактна в топологии $\Xi$.

Рассмотрим основные примеры трансляционно компактных функций, которые будут использоваться в данной статье.

Пример 2.1. Пусть $\Xi=C_{\mathrm{b}}(\mathbb{R} ; \mathscr{M})$, где $\mathscr{M}$ - полное метрическое пространство. Пусть $\sigma_{0}(s)$ - почти периодическая функция со значениями в $\mathscr{M}$. Из критерия Бохнера-Америо известно, что почти периодическая функция $\sigma_{0}(s)$ обладает следующим характеристическим свойством: множество ее трансляций $\left\{\sigma_{0}(s+h)=T(h) \sigma_{0}(s) \mid h \in \mathbb{R}\right\}$ образует предкомпактное множество в $C_{\mathrm{b}}(\mathbb{R} ; \mathscr{M})$ (см., например, [16], [17]). Замыкание в $C_{\mathrm{b}}(\mathbb{R} ; \mathscr{M})$ этого множества служит оболочкой $\mathscr{H}\left(\sigma_{0}\right)$ функции $\sigma_{0}(s)$ (см. $\left.(2.9)\right)$. Значит, по определению $2.2, \sigma_{0}(s)$ является трансляционно компактной функцией в $C_{\mathrm{b}}(\mathbb{R} ; \mathscr{M})$. Если функция $\sigma_{0}(s)$ является почти периодической, то любая функция $\sigma(s) \in \mathscr{H}\left(\sigma_{0}\right)$ также является почти периодической. Легко видеть, что группа трансляций $\{T(h) \mid h \in \mathbb{R}\}$ непрерывна в $C_{\mathrm{b}}(\mathbb{R} ; \mathscr{M})$.

Важным подклассом почти периодических функций являются квазипериодические функции, которые представимы в виде

$$
\sigma_{0}(s)=\phi\left(\alpha_{1} s, \alpha_{2} s, \ldots, \alpha_{k} s\right)=\phi(\bar{\alpha} s), \quad \bar{\alpha}=\left(\alpha_{1}, \alpha_{2}, \ldots, \alpha_{k}\right) \in \mathbb{R}^{k},
$$


где функция $\phi(\bar{\omega})=\phi\left(\omega_{1}, \omega_{2}, \ldots, \omega_{k}\right)$ является непрерывной и $2 \pi$-периодической по каждому аргументу $\omega_{i} \in \mathbb{R}$. Пусть $\mathbb{T}^{k}=[\mathbb{R} \bmod 2 \pi]^{k}$ обозначает $k$-мерный тор. Тогда $\phi \in C\left(\mathbb{T}^{k} ; \mathscr{M}\right)$. Предполагается, что вещественные числа $\alpha_{1}, \alpha_{2}, \ldots, \alpha_{k}$ в (2.10) являются рационально независимыми. Оболочка $\mathscr{H}\left(\sigma_{0}\right)$ квазипериодической функции $\sigma_{0}(s)$ в пространстве $C_{\mathrm{b}}(\mathbb{R} ; \mathscr{M})$ совпадает с $\left\{\phi\left(\bar{\alpha} s+\bar{\omega}_{1}\right) \mid \bar{\omega}_{1} \in \mathbb{T}^{k}\right\}=\mathscr{H}\left(\sigma_{0}\right)$. При $k=1$ получаются периодические функции: $\sigma_{0}(s+2 \pi)=\sigma_{0}(s)$.

Аналогично рассматриваются трансляционно компактные функции на полуоси в пространстве $\Xi_{+}=C_{\mathrm{b}}\left(\mathbb{R}_{+} ; \mathscr{M}\right)$, для которых оболочка $\left\{\sigma_{0}(s+h)=\right.$ $\left.T(h) \sigma_{0}(s) \mid h \geqslant 0\right\}$ предкомпактна в $C_{\mathrm{b}}\left(\mathbb{R}_{+} ; \mathscr{M}\right)$. Такие трансляционно компактные функции иногда называют асимптотически почти периодическими.

В [7] приведены другие примеры трансляционно компактных функций, которые не являются квазипериодическими или почти периодическими.

ПРИмеР 2.2. Пусть $\Xi=L_{p}^{\text {loc }}(\mathscr{R} ; \mathscr{E})$, где $p \geqslant 1$ и $\mathscr{E}-$ пространство Банаха. Пространство $L_{p}^{\operatorname{loc}}(\mathscr{R} ; \mathscr{E})$ состоит из функций $\xi(t), t \in \mathscr{R}$, со значениями в $\mathscr{E}$, которые являются локально интегрируемыми по Бохнеру в степени $p$, т. е.

$$
\int_{t_{1}}^{t_{2}}\|\xi(s)\|_{\mathscr{E}}^{p} d s<+\infty \quad \forall\left[t_{1}, t_{2}\right] \subset \mathscr{R} \text {. }
$$

Рассматривается следующая топология локальной сходимости в пространстве $L_{p}^{\mathrm{loc}}(\mathscr{R} ; \mathscr{E})$. По определению, $\xi_{n}(s) \rightarrow \xi(s)(n \rightarrow \infty)$ в $L_{p}^{\mathrm{loc}}(\mathscr{R} ; \mathscr{E})$, если

$$
\int_{t_{1}}^{t_{2}}\left\|\xi_{n}(s)-\xi(s)\right\|_{\mathscr{E}}^{p} d s \rightarrow 0 \quad(n \rightarrow \infty)
$$

для каждого отрезка $\left[t_{1}, t_{2}\right] \subset \mathscr{R} . \quad$ Пространство $L_{p}^{\text {loc }}(\mathscr{R} ; \mathscr{E})$ является счетно нормируемым, метризуемым и полным. Рассмотрим трансляционно компактные функции в пространстве $L_{p}^{\text {loc }}(\mathscr{R} ; \mathscr{E})$. Имеет место следующий критерий (см., например, [7]): функция $\sigma_{0}(s)$ является трансляционно компактной в $L_{p}^{\text {loc }}(\mathscr{R} ; \mathscr{E})$ тогда и только тогда, когда (i) для любого $h \geqslant 0$ множество $\left\{\int_{t}^{t+h} \sigma_{0}(s) d s \mid t \in \mathscr{R}\right\}$ предкомпактно в $\mathscr{E}$ и (iі) найдется положительная функция $\beta(s) \rightarrow 0(s \rightarrow 0+)$ такая, что

$$
\int_{t}^{t+1}\left\|\sigma_{0}(s)-\sigma_{0}(s+l)\right\|_{\mathscr{E}}^{p} d s \leqslant \beta(|l|) \quad \forall t \in \mathscr{R}
$$

Из этого критерия следует, что

$$
\sup _{t \in \mathscr{R}} \int_{t}^{t+1}\left\|\sigma_{0}(s)\right\|_{\mathscr{E}}^{p} d s<+\infty
$$

для любой трансляционно компактной функции в $L_{p}^{\mathrm{loc}}(\mathscr{R} ; \mathscr{E})$. Очевидно, что полугруппа (или группа) трансляций $\{T(h) \mid h \in \mathscr{R}\}$ непрерывна в $L_{p}^{\text {loc }}(\mathscr{R} ; \mathscr{E})$. 
ПримеР 2.3. Аналогично вводятся трансляционно компактные функции в пространстве $\Xi=C^{\text {loc }}(\mathscr{R} ; \mathscr{M})$, которое состоит из непрерывных функций $\xi(s)$, $s \in \mathscr{R}$, со значениями в $\mathscr{M}$. Топология в $C^{\text {loc }}(\mathscr{R} ; \mathscr{M})$ порождается топологией локальной равномерной сходимости на каждом интервале $\left[t_{1}, t_{2}\right] \subset \mathscr{R}($ см. [7]). Из теоремы Арцела-Асколи о компактности вытекает следующий критерий: функция $\sigma_{0}(s)$ является трансляционно компактной в $C^{\text {loc }}(\mathscr{R} ; \mathscr{M})$ тогда и только тогда, когда (i) множество $\left\{\sigma_{0}(h) h \in \mathscr{R}\right\}$ предкомпактно в $\mathscr{E}$ и (ii) $\sigma_{0}(s)$ равномерно непрерывна на $\mathscr{R}$, т. е. найдется положительная функция $\alpha(s) \rightarrow 0+$ $(s \rightarrow 0+)$ такая, что

$$
\left\|\sigma_{0}\left(s_{1}\right)-\sigma_{0}\left(s_{2}\right)\right\|_{\mathscr{E}} \leqslant \alpha\left(\left|s_{1}-s_{2}\right|\right) \quad \forall s_{1}, s_{2} \in \mathscr{R}
$$

(см. подробности в [7]). В частности, любая трансляционно компактная функция в $C^{\operatorname{loc}}(\mathscr{R} ; \mathscr{M})$ ограничена в $\mathscr{M}$. Полугруппа трансляций $\{T(h) \mid h \in \mathscr{R}\}$, очевидно, непрерывна в $C^{\text {loc }}(\mathscr{R} ; \mathscr{M})$.

Легко видеть, что почти периодические функции со значениями в $\mathscr{M}$, т. е. трансляционно компактные в $C_{\mathrm{b}}(\mathbb{R} ; \mathscr{M})$, являются также трансляционно компактными в пространстве $C^{\mathrm{loc}}(\mathbb{R} ; \mathscr{M})$.

При $\mathscr{R}=\mathbb{R}$ оболочки $\mathscr{H}\left(\sigma_{0}\right)=\Sigma$ всех рассмотренных классов трансляционно компактных функций являются строго инвариантными множествами для группы трансляций $\{T(h), h \in \mathbb{R}\}$, т. е. выполнено свойство $(2.6)$.

При $\mathscr{R}=\mathbb{R}_{+}$соответствующие оболочки $\Sigma$ удовлетворяют (2.7). Оболочки трансляционно компактных функций на полуоси мы будем иногда обозначать $\mathscr{H}_{+}\left(\sigma_{0}\right)$, чтобы отличать их от оболочек трансляционно компактных функций на всей оси. Такие трансляционно компактные функции будут использоваться при изучении полупроцессов.

Рассмотрим частный случай временно́го символа $\sigma_{0}(s)$ уравнения $(2.4)$, когда трансляции $\{T(h) \mid h \geqslant 0\}$ отображают его на себя: $T(h) \sigma_{0}(s)=\sigma_{0}(s+h) \equiv$ $\sigma_{0}(s)$ при всех $h \geqslant 0$, т. е. $\sigma_{0}(t)$ не зависит от времени: $\sigma_{0}(s)=\sigma_{0}$ при всех $s \in \mathscr{R}$, где $\sigma_{0} \in \mathscr{M}$. Тогда в силу $(2.8)$ соответствующий $\mathscr{R}$-процесс $\left\{U_{\sigma_{0}}(t, \tau)\right\}$ удовлетворяет тождеству $U_{\sigma_{0}}(t, \tau)=U_{\sigma_{0}}(t+h, \tau+h)=U_{\sigma_{0}}(t-\tau, 0)$ при всех $h \geqslant 0, t \geqslant \tau, \tau \in \mathscr{R}$. Следовательно, процесс $\left\{U_{\sigma_{0}}(t, \tau)\right\}$ полностью описывается однопараметрическим семейством отображений $S(t)=U_{\sigma_{0}}(t, 0), t \geqslant 0$. Легко видеть, что $\{S(t)\}$ образуют динамическую полугруппу, которая отвечает автономному уравнению (2.4) с постоянным символом $\sigma(t)=\sigma_{0}$.

Напомним, что семейство отображений $\{S(t), t \geqslant 0\}, S(t): E \rightarrow E$, называется полугруппой, если $S(0)=\mathrm{Id}$ - тождественное отображение и выполнено соотношение

$$
S\left(t_{1}+t_{2}\right)=S\left(t_{1}\right) \circ S\left(t_{2}\right) \quad \forall t_{1}, t_{2} \geqslant 0 .
$$

Изучению аттракторов диссипативных динамических систем, которые порождаются автономными уравнениями, посвящена обширная литература (см. [1]-[5], [8]). Заметим, что полугруппы, порождаемые автономными эволюционными уравнениями, являются частным случаем процессов, порождаемых неавтономными уравнениями. 
В завершение этого раздела сформулируем теорему о существовании глобальных аттракторов асимптотически компактных полугрупп, которая будет использоваться в дальнейшем. В этой формулировке традиционное условие непрерывности полугруппы заменено на более слабое условие ее замкнутости. В таком виде эта общая теорема доказана в [18] (см. также [19]).

Теорема 2.1. Если полугруппа $\{S(t)\}$, действующая в полном метрическом пространстве E, является асимптотически компактной, то у нее имеется компактный глобальный аттрактор $\mathscr{A}$. Если дополнительно известно, что полугруппа $\{S(t)\}$ замкнута в $E$, то $\mathscr{A}$ строго инвариантно, т.е. $S(t) \mathscr{A}=\mathscr{A}$ при всех $t \geqslant 0$.

Отметим, что для полугрупп, действующих в компактных пространствах, замкнутость эквивалентна непрерывности.

\section{3. Сведение к полугруппе в расширенном фазовом пространстве. Косое произведение}

Пусть в полном метрическом или банаховом пространстве $E$ действует семейство $\mathscr{R}$-процессов $\left\{U_{\sigma}(t, \tau)\right\}, \sigma \in \Sigma$, причем пространство символов $\Sigma$ является метрическим и полным. Пусть на $\Sigma$ действует некоторая полугруппа $\{T(h)\}_{h \geqslant 0}: T(h) \Sigma \subseteq \Sigma$ при $h \geqslant 0$ (в случае $\mathscr{R}=\mathbb{R}$ эта полугруппа может быть группой, но в общем случае это не обязательно).

Здесь не требуется, чтобы $\Sigma$ было оболочкой некой трансляционно компактной функции, и также не предполагается, что $\{T(h)\}$ является полугруппой трансляций по времени в пространстве $\Xi=\{\xi(t) \mid t \in \mathscr{R}\}$ (или $\left.C_{\mathrm{b}}(\mathscr{R} ; \mathscr{M})\right)$, как это было в разделе 2 .

Предполагается выполненным следующее трансляиионное тождество:

$$
U_{\sigma}(t+h, \tau+h)=U_{T(h) \sigma}(t, \tau) \quad \forall \sigma \in \Sigma, \quad t \geqslant \tau, \quad \tau \in \mathscr{R}, \quad h \geqslant 0 .
$$

Тождество (3.1) аналогично тождеству (2.8), которое выполнено для рассмотренной в разделе 2 полугруппы трансляций.

При выполнении условия (3.1) построим полугруппу $\{\mathscr{S}(t) \mid t \geqslant 0\}$, действующую в расширенном фазовом пространстве $E \times \Sigma$, которое соответствует рассматриваемому семейству $\mathscr{R}$-процессов $\left\{U_{\sigma}(t, \tau)\right\}, \sigma \in \Sigma$. Рассмотрим следующие отображения в $E \times \Sigma$ :

$$
\mathscr{S}(t)(u, \sigma)=\left(U_{\sigma}(t, 0) u, T(t) \sigma\right), \quad t \geqslant 0, \quad(u, \sigma) \in E \times \Sigma .
$$

УтВЕРЖДЕНИЕ 3.1. Если выполнено условие (3.1), то семейство отображений $\{\mathscr{S}(t)\}$, действующих в $E \times \Sigma$ по формуле (3.2), образует полугруппу в $E \times \Sigma$.

ДокАЗАтЕльство. Достаточно проверить полугрупповое соотношение:

$$
\begin{aligned}
\mathscr{S}\left(t_{1}+t_{2}\right)(u, \sigma) & =\left(U_{\sigma}\left(t_{1}+t_{2}, 0\right) u, T\left(t_{1}+t_{2}\right) \sigma\right) \\
& =\left(U_{\sigma}\left(t_{1}+t_{2}, t_{2}\right) U_{\sigma}\left(t_{2}, 0\right) u, T\left(t_{1}\right) T\left(t_{2}\right) \sigma\right) \\
& =\left(U_{T\left(t_{2}\right) \sigma}\left(t_{1}, 0\right) U_{\sigma}\left(t_{2}, 0\right) u, T\left(t_{1}\right)\left(T\left(t_{2}\right) \sigma\right)\right) \\
& =\mathscr{S}\left(t_{1}\right)\left(U_{\sigma}\left(t_{2}, 0\right) u, T\left(t_{2}\right) \sigma\right)=\mathscr{S}\left(t_{1}\right) \mathscr{S}\left(t_{2}\right)(u, \sigma) .
\end{aligned}
$$


Здесь использовалось свойство (1.2) R्R-процесса, а также трансляционное тождество (3.1). Очевидно, что $\mathscr{S}(0)=\mathrm{Id}-$ тождественное отображение. Утверждение доказано.

Конструкцию (3.2) часто называют косым произведением (см. [3], [8]).

Рассмотрим некоторые примеры построения полугрупп в расширенном пространстве.

Пример 3.1 (любой процесс). Пусть $\{U(t, \tau)\}$ - произвольный $\mathscr{R}$-процесс. Рассматривается семейство $\mathscr{R}$-процессов $\left\{U_{\sigma}(t, \tau)\right\}, \sigma \in \Sigma$, задаваемое формулой $U_{\sigma}(t, \tau)=U(t+\sigma, \tau+\sigma)$, где пространство символов $\Sigma$ совпадает с $\mathscr{R}$. Рассмотрим семейство трансляций $T(h) \sigma=h+\sigma, h \geqslant 0$, которые образуют полугруппу в $\mathscr{R}$. Свойство (3.1) легко проверяется:

$U_{\sigma}(t+h, \tau+h)=U(t+h+\sigma, \tau+h+\sigma)=U(t+T(h) \sigma, \tau+T(h) \sigma)=U_{T(h) \sigma}(t, \tau)$.

Полугруппа $\{\mathscr{S}(t)\}$ на $E \times \mathscr{R}$ действует по формуле (3.2). Это простейший способ сведения неавтономной динамической системы к автономной. Однако у такой полугруппы не может быть компактного (и даже ограниченного) притягивающего или поглощающего множества в расширенном фазовом пространстве $E \times \mathscr{R}$.

ПРимеР 3.2 (периодический прощесс). Пусть $\{U(t, \tau)\}$ - периодический процесс с периодом $p>0(\mathscr{R}=\mathbb{R})$, т. е.

$$
U(t+p, \tau+p)=U(t, \tau) \quad \forall t \geqslant \tau, \quad \tau \in \mathbb{R} .
$$

Рассмотрим окружность $\Sigma=\mathbb{T}^{1}=\mathbb{R}(\bmod p)$ в качестве пространства символов. Как и в примере 3.1 , зададим семейство $\left\{U_{\sigma}(t, \tau)\right\}, \sigma \in \mathbb{T}^{1}$, формулой $U_{\sigma}(t, \tau)=U(t+\sigma, \tau+\sigma)$. Полугруппа $\{T(h)\}$ состоит из вращений окружности: $T(h) \sigma=h+\sigma(\bmod p)$. Очевидно, что тождество $(3.1)$ выполнено. Формула (3.2) задает полугруппу $\{\mathscr{S}(t)\}$ в $E \times \mathbb{T}^{1}$. Заметим, что если семейство процессов $\left\{U_{\sigma}(t, \tau)\right\}, \sigma \in \mathbb{T}^{1}$, имеет компактное равномерно притягивающее множество $P$, то у полугруппы $\{\mathscr{S}(t)\}$ также имеется компактное притягивающее множество $P \times \mathbb{T}^{1}$ в расширенном фазовом пространстве $E \times \mathscr{R}$.

ПримеР 3.3 (неавтономное уравнение с транслячионно компактным символом $\left.\sigma_{0}\right)$. Этот пример обсуждался в разделе 2. Задача $(2.4)-(2.5)$ порождает семейство $\mathscr{R}$-процессов $\left\{U_{\sigma}(t, \tau)\right\}, \sigma \in \Sigma=\mathscr{H}\left(\sigma_{0}\right)$, где $\mathscr{H}\left(\sigma_{0}\right)$ - оболочка трансляционно компактной функции $\sigma_{0}(s)$ в $\Xi$. Пространство символов $\Sigma=\mathscr{H}\left(\sigma_{0}\right)$ компактно, и на нем действует трансляционная полугруппа $\{T(h)\}$ : $T(h) \sigma(s)=\sigma(s+h), \sigma \in \mathscr{H}\left(\sigma_{0}\right), T(h) \mathscr{H}\left(\sigma_{0}\right) \subseteq \mathscr{H}\left(\sigma_{0}\right)$ при $h \geqslant 0$. Трансляционное тождество выполнено, и с помощью утверждения 3.1 строится косое произведение $\{\mathscr{S}(t)\}$, действующее в $E \times \mathscr{H}\left(\sigma_{0}\right)$ по формуле

$$
\mathscr{S}(t)\left(u_{0}, \sigma\right)=\left(U_{\sigma}(t, 0) u_{0}, \sigma(t+s)\right), \quad u_{0} \in E, \quad \sigma \in \mathscr{H}\left(\sigma_{0}\right),
$$

где $U_{\sigma}(t, 0) u_{0}=u(t)$ - решение $(2.4)$ с символом $\sigma(s)$ и начальными данными $\left.u\right|_{t=0}=u_{0} \in E$. В частности, можно рассмотреть $\Xi=C_{\mathrm{b}}(\mathbb{R} ; \mathscr{M})$ и уравнение $(2.4)$ с пространством символов $\Sigma=\mathscr{H}\left(\sigma_{0}\right)$, где $\sigma_{0}(s)$ - почти периодическая функция со значениями в $\mathscr{M}$. 
ПримеР 3.4 (неавтономное уравнение с квазипериодическим символом). Пусть в предыдущем примере

$$
\sigma_{0}(s)=\varphi\left(\alpha_{1} s, \ldots, \alpha_{k} s\right)=\varphi(\boldsymbol{\alpha} s), \quad s \in \mathbb{R},
$$

где $\boldsymbol{\alpha}=\left(\alpha_{1}, \ldots, \alpha_{k}\right) \in \mathbb{R}_{+}^{k}, \varphi\left(\omega_{1}, \ldots, \omega_{k}\right)-2 \pi$-периодическая функция по каждому аргументу $\omega_{i}, \boldsymbol{\omega}=\left(\omega_{1}, \ldots, \omega_{k}\right) \in \mathbb{T}^{k}, \varphi \in C\left(\mathbb{T}^{k} ; \mathscr{M}\right)$. Предполагается, что числа $\left\{\alpha_{i} \mid i=1, \ldots, k\right\}$ рационально независимы. Рассмотрим оболочку $\Sigma=\mathscr{H}\left(\sigma_{0}\right)$. В примере 2.1 показано, что $\mathscr{H}\left(\sigma_{0}\right)=\left\{\varphi\left(\boldsymbol{\alpha} s+\boldsymbol{\omega}_{0}\right) \mid \boldsymbol{\omega}_{0} \in \mathbb{T}^{k}\right\}$. Можно рассмотреть пространство символов $\Sigma=\mathbb{T}^{k}$ вместо $\mathscr{H}\left(\sigma_{0}\right)$, имея в виду непрерывное отображение из $\mathbb{T}^{k}$ в $\mathscr{H}\left(\sigma_{0}\right): \boldsymbol{\omega}_{0} \mapsto \varphi\left(\boldsymbol{\alpha} t+\boldsymbol{\omega}_{0}\right)$. Трансляционной полугруппе на $\mathscr{H}\left(\sigma_{0}\right)$ соответствует полугруппа трансляций на торе $\mathbb{T}^{k}$, $T(h) \boldsymbol{\omega}_{0}=\left[\boldsymbol{\omega}_{0}+\boldsymbol{\alpha} h\right]=\boldsymbol{\omega}_{0}+\boldsymbol{\alpha} h(\bmod 2 \pi)^{k}$. Очевидно, что $T(h) \mathbb{T}^{k}=\mathbb{T}^{k}$. Косое произведение $\{\mathscr{S}(t)\}$ действует на $E \times \mathbb{T}^{k}$ по формуле

$$
\mathscr{S}(t)\left(u_{0}, \boldsymbol{\omega}_{0}\right)=\left(U_{\boldsymbol{\omega}_{0}}(t, 0) u_{0},\left[\boldsymbol{\omega}_{0}+\boldsymbol{\alpha} t\right]\right), \quad u_{0} \in E, \quad \boldsymbol{\omega}_{0} \in \mathbb{T}^{k},
$$

где $u(t)=U_{\boldsymbol{\omega}_{0}}(t, 0) u_{0}=U_{\sigma}(t, 0) u_{0}$ - решение $(2.4)$ с квазипериодическим символом $\sigma(s)=\varphi\left(\boldsymbol{\alpha} s+\boldsymbol{\omega}_{0}\right)$ и с начальным условием $\left.u\right|_{t=0}=u_{0} \in E$.

Вернемся к изучению общих семейств процессов.

ОПРеДЕЛЕНиЕ 3.1. Семейство $\mathscr{R}$-процессов $\left\{U_{\sigma}(t, \tau)\right\}, \sigma \in \Sigma$, называется $(E \times \Sigma, E)$-непрерывным, если при любых фиксированных $t$ и $\tau, t \geqslant \tau$, отображение $(u, \sigma) \mapsto U_{\sigma}(t, \tau) u$ непрерывно из $E \times \Sigma$ в $E$.

Семейство $\mathscr{R}$-процессов $\left\{U_{\sigma}(t, \tau)\right\}, \sigma \in \Sigma$, называется $(E \times \Sigma, E)$-замкнутым, если при любых фиксированных $t$ и $\tau, t \geqslant \tau$, отображение $(u, \sigma) \mapsto U_{\sigma}(t, \tau) u$ из $E \times \Sigma$ в $E$ замкнуто, т. е. замкнут график этой функции в $(E \times \Sigma) \times E$. Как известно, замкнутость следует из непрерывности, но не наоборот.

Рассмотрим два проектора $\Pi_{1}$ и $\Pi_{2}$ из $E \times \Sigma$ в $E$ и $\Sigma$ соответственно:

$$
\Pi_{1}(u, \sigma)=u, \quad \Pi_{2}(u, \sigma)=\sigma .
$$

Сформулируем основную теорему об аттракторах семейств $\mathscr{R}$-процессов.

Теорема 3.1. Пусть семейство $\mathscr{R}$-процессов $\left\{U_{\sigma}(t, \tau)\right\}, \sigma \in \Sigma$, действующих в пространстве $E$, является равномерно асимптотически компактным $u(E \times \Sigma, E)$-замкнутым. Пусть $\Sigma$ - ограниченное полное метрическое пространство, на котором действует замкнутая полугруппа $\{T(h)\}$, удовлетворяющая трансляционному тождеству (3.1). Тогда полугруппа $\{\mathscr{S}(t)\}$, действующая в расширенном фазовом пространстве $E \times \Sigma$ по формуле (3.2), имеет компактный аттрактор $\mathbf{A}$, который строго инвариантен относительно $\{\mathscr{S}(t)\}, \mathscr{S}(t) \mathbf{A}=\mathbf{A}$ при всех $t \geqslant 0$. Кроме того,

(i) множество $\mathbf{A}_{1}:=\Pi_{1} \mathbf{A}=\mathscr{A}_{\Sigma}$ является равномерным (по $\sigma \in \Sigma$ ) глобальным аттрактором семейства $\mathscr{R}$-процессов $\left\{U_{\sigma}(t, \tau)\right\}, \sigma \in \Sigma$;

(ii) множество $\mathbf{A}_{2}:=\Pi_{2} \mathbf{A}=\omega(\Sigma)$ является глобальным аттрактором полугруппь $\{T(h)\}$, действующей на $\Sigma: T(h) \omega(\Sigma)=\omega(\Sigma)$ при всех $h \geqslant 0$;

(iii) множество $\mathscr{A}_{\Sigma}=\mathscr{A}_{\omega(\Sigma)}$ является такюе равномерным $($ по $\sigma \in \omega(\Sigma))$ глобальным аттрактором семейства $\mathscr{R}$-процессов $\left\{U_{\sigma}(t, \tau)\right\}, \sigma \in \omega(\Sigma)$. 
Сформулированная теорема является обобщением аналогичной теоремы, доказанной, например, в [7]. Существенное отличие заключается в том, что в новой теореме условие непрерывности семейства $\mathscr{R}$-процессов заменено на более слабое условие замкнутости семейства $\mathscr{R}$-процессов. При этом используется модифицированная теорема 2.1. Доказательство теоремы 3.1 в основных чертах совпадает с доказательством из [7], поэтому мы его здесь не приводим.

ЗАмечАниЕ 3.1. Стоит обратить внимание на совпадение аттракторов $\mathscr{A}_{\Sigma}=$ $\mathscr{A}_{\omega(\Sigma)}$ двух вложенных семейств $\mathscr{R}$-процессов $\left\{U_{\sigma}(t, \tau)\right\}$ при $\sigma \in \Sigma$ и при $\sigma \in \omega(\Sigma)$. В общем случае $\omega(\Sigma) \subset \Sigma$ и следствие 1.1 дает лишь соотношение $\mathscr{A}_{\Sigma} \supseteq \mathscr{A}_{\omega(\Sigma)}$.

\section{4. О структуре равномерных глобальных аттракторов семейств процессов. Приложения к УрЧП}

В этом разделе будут изучаться семейства процессов $\left\{U_{\sigma}(t, \tau)\right\}, \sigma \in \Sigma$, т. е. когда $\mathscr{R}=\mathbb{R}$. Будет предложено простое описание для равномерных глобальных аттракторов семейств процессов с помощью сечений их ядер, которое будет использоваться в дальнейших разделах.

Введем понятие полной траектории процесса, которое участвует в определении ядра процесса. Функция $u(s), s \in \mathbb{R}$, со значениями в $E$ называется полной траекторией процесса $\{U(t, \tau)\}$, если

$$
U(t, \tau) u(\tau)=u(t) \quad \text { при всех } t \geqslant \tau, \tau \in \mathbb{R} .
$$

Полная траектория $u(s)$ называется ограниченной, если множество $\{u(s), s \in \mathbb{R}\}$ ограничено в $E$.

ОПРЕДЕЛЕНИЕ 4.1. Ядро $\mathscr{K}$ процесса $\{U(t, \tau)\}$ есть объединение всех ограниченных полных траекторий этого процесса:

$$
\mathscr{K}=\left\{u(\cdot) \mid u \text { удовлетворяет (4.1) и }\|u(s)\|_{E} \leqslant C_{u} \forall s \in \mathbb{R}\right\} .
$$

Множество

$$
\mathscr{K}(t)=\{u(t) \mid u(\cdot) \in \mathscr{K}\} \subset E, \quad t \in \mathbb{R},
$$

называется сечением ядра процесса $\{U(t, \tau)\}$ в момент $t$.

В общем случае сечения ядра $\mathscr{K}(t)$ зависят от времени. Легко проверить следующее утверждение.

УТВЕРЖДЕНИЕ 4.1. Если семейство процессов $\left\{U_{\sigma}(t, \tau)\right\}, \sigma \in \Sigma$, имеет равномерный (по $\sigma \in \Sigma)$ глобальный аттрактор $\mathscr{A}_{\Sigma}$, то

$$
\bigcup_{\sigma \in \Sigma} \bigcup_{t \in \mathbb{R}} \mathscr{K}_{\sigma}(t) \subseteq \mathscr{A}_{\Sigma} .
$$

Цель данного раздела - установить достаточные условия, при которых в соотношении (4.2) можно поставить знак равенства. 
Сформулируем уточнение теоремы 3.1 для случая семейств процессов.

Теорема 4.1. Пусть семейство процессов $\left\{U_{\sigma}(t, \tau)\right\}, \sigma \in \Sigma$, является равномерно асимптотически компактным и $(E \times \Sigma, E)$-замкнутым. Предполагается, что $\Sigma$ - компактное метрическое пространство, на котором действует замкнутая полугруппа $\{T(h)\}$, удовлетворяющая транслячионному тождеству (3.1). Пусть $\Sigma$ строго инвариантно относительно $\{T(h)\}: T(h) \Sigma=\Sigma$ при всех $h \geqslant 0$. Тогда полугруппа $\{\mathscr{S}(t)\}$, действующая в расширенном пространстве $E \times \Sigma$ по формуле (3.2), имеет компактный глобальный аттрактор $\mathbf{A}$, причем $\mathscr{S}(t) \mathbf{A}=\mathbf{A}$ при всех $t \geqslant 0$. Более того,

(i) множество $\mathbf{A}_{1}:=\Pi_{1} \mathbf{A}=\mathscr{A}_{\Sigma}$ является равномерным (по $\sigma \in \Sigma$ ) глобальным аттрактором семейства $\mathscr{R}$-процессов $\left\{U_{\sigma}(t, \tau)\right\}, \sigma \in \Sigma$;

(ii) множество $\mathbf{A}_{2}:=\Pi_{2} \mathbf{A}$ совпадает с $\Sigma$;

(iii) равномерный глобальный аттрактор $\mathscr{A}_{\Sigma}$ удовлетворяет равенству

$$
\mathscr{A}_{\Sigma}=\bigcup_{\sigma \in \Sigma} \mathscr{K}_{\sigma}(0) .
$$

Отметим, что момент времени $t=0$ в утверждении (iii) теоремы 4.1 можно заменить на любой другой момент $t=\theta$, т. е. в формуле (4.2) получаем равенство. Доказательство пункта (iii) приведено в [7]. Из пунктов (ii) и (iii) вытекает, что ядро $\mathscr{K}_{\sigma}$ непусто при любом $\sigma \in \Sigma$.

Сформулируем этот результат в том виде, в котором его будет удобно применять к важным частным случаям, изложенным в разделе 2 , а также в примерах 3.3 и 3.4, которые относятся к исследованию равномерных аттракторов неавтономных уравнений вида $(2.4)$ на всей оси времени $\mathbb{R}$.

СлеДСТВИЕ 4.1. Пусть $\sigma_{0}(t)$ - трансляиионно компактная (или почти периодическая, или квазипериодическая) функиия $u\left\{U_{\sigma}(t, \tau)\right\}, \sigma \in \mathscr{H}\left(\sigma_{0}\right),-$-емейство прочессов, порожденное неавтономной задачей (2.4)-(2.5), m.е. пространство символов $\Sigma$ совпадает с $\mathscr{H}\left(\sigma_{0}\right)$. Предположим, что семейство $\left\{U_{\sigma}(t, \tau)\right\}, \sigma \in \mathscr{H}\left(\sigma_{0}\right)$, является равномерно асимптотически компактным и $\left(E \times \mathscr{H}\left(\sigma_{0}\right), E\right)$-замкнутым. Тогда множество

$$
\mathscr{A}_{\mathscr{H}\left(\sigma_{0}\right)}:=\bigcup_{\sigma \in \mathscr{H}\left(\sigma_{0}\right)} \mathscr{K}_{\sigma}(0)
$$

есть равномерный глобалъный аттрактор семейства прочессов $\left\{U_{\sigma}(t, \tau)\right\}, \sigma \in$ $\mathscr{H}\left(\sigma_{0}\right)$, где $\mathscr{K}_{\sigma}(0)$ - сечение ядра $\mathscr{K}_{\sigma}$ прощесса $\left\{U_{\sigma}(t, \tau)\right\}$ в момент времени $t=0$. Ядро $\mathscr{K}_{\sigma}$ непусто при любом $\sigma \in \mathscr{H}\left(\sigma_{0}\right)$.

Рассмотрим некоторые важные примеры неавтономных уравнений математической физики, для которых будут построены равномерные глобальные аттракторы с помощью приведенных выше результатов.

Пример 4.1 (2D-система Навъе-Стокса с зависящей от времени внешней силой). Эта система описывает плоское течение вязкой несжимаемой жидкости в ограниченной области $\Omega \Subset \mathbb{R}^{2}$ с граничными условиями прилипания. После исключения функции давления система записывается в виде

$$
\begin{aligned}
& \partial_{t} u+\nu L u+B(u, u)=g(x, t), \quad t \in \mathbb{R}, \\
& \left.u\right|_{\partial \Omega}=0, \quad x=\left(x_{1}, x_{2}\right) \in \Omega,
\end{aligned}
$$


где неизвестной служит вектор-функция $u=u(x, t)=\left(u^{1}(x, t), u^{2}(x, t)\right)$, а зависящая от времени внешняя сила $g(x, t)=\left(g^{1}(x, t), g^{2}(x, t)\right)$ является известной. Задан также коэффициент вязкости $\nu>0$ (см. [20], [12], [21], [22], [1]).

Обозначим через $H$ и $H_{1}$ замыкание по нормам | | | и $\|$. | пространств $\left(L_{2}(\Omega)\right)^{2}$ и $\left(H_{0}^{1}(\Omega)\right)^{2}$ множества двумерных векторных функций $\mathscr{V}_{0}=\{v \in$ $\left.\left(C_{0}^{\infty}(\Omega)\right)^{2}, \nabla \cdot v=0\right\}$. Через $P$ обозначается ортогональный проектор из $\left(L_{2}(\Omega)\right)^{2}$ на подпространство $H$. В уравнении (4.3)

$$
L=-\Pi \Delta, \quad B(u, u)=\Pi \sum_{i=1}^{2} u^{i} \partial_{x_{i}} u .
$$

Известно, что оператор Стокса $L$ с областью определения $H^{1} \cap\left(H^{2}(\Omega)\right)^{2}$ является самосопряженным и положительным. Пусть $\lambda_{1}-$ первое собственное значение $L$.

Начальные условия ставятся при $t=\tau, \tau \in \mathbb{R}$ :

$$
\left.u\right|_{t=\tau}=u_{\tau}, \quad u_{\tau} \in H .
$$

Система (4.3) является неавтономной, ее временнь́м символом $\sigma(s), s \in \mathbb{R}$, служит внешняя сила $g(s)$. Предполагается, что функция $g(s):=g(\cdot, s)$ принадлежит $L_{2}^{\text {loc }}(\mathbb{R} ; H)$ и является ограниченной в $L_{2}^{\mathrm{b}}(\mathbb{R} ; H)$, т. е.

$$
\|g\|_{L_{2}^{\mathrm{b}}}^{2}=\|g\|_{L_{2}^{\mathrm{b}}(\mathbb{R} ; H)}^{2}=\sup _{h \in \mathbb{R}} \int_{h}^{h+1}|g(s)|^{2} d s<\infty .
$$

Задача (4.3)-(4.4) имеет единственное решение $u(\cdot) \in C\left(\mathbb{R}_{\tau} ; H\right) \cap L_{2}^{\text {loc }}\left(\mathbb{R}_{\tau} ; H^{1}\right)$, причем $\partial_{t} u(\cdot) \in L_{2}^{\text {loc }}\left(\mathbb{R}_{\tau} ; H^{-1}\right)$, где $\mathbb{R}_{\tau}=[\tau,+\infty), H^{-1}=\left(H^{1}\right)^{*}$. Имеют место следующие оценки:

$$
\begin{gathered}
|u(t)|^{2} \leqslant|u(\tau)|^{2} e^{-\lambda(t-\tau)}+\lambda^{-1}\left(1+\lambda^{-1}\right)\|g\|_{L_{2}^{\mathrm{b}}}^{2}, \\
|u(t)|^{2}+\nu \int_{\tau}^{t}\|u(s)\|^{2} d s \leqslant|u(\tau)|^{2}+\lambda^{-1} \int_{\tau}^{t}|g(s)|^{2} d s, \quad \lambda=\nu \lambda_{1}, \\
(t-\tau)\|u(t)\|^{2} \leqslant C\left(t-\tau,|u(\tau)|^{2}, \int_{\tau}^{t}|g(s)|^{2} d s\right),
\end{gathered}
$$

где $C\left(z, R, R_{1}\right)$ - монотонная функция от $z=t-\tau, R$ и $R_{1}$. Доказательство можно найти в книгах, упомянутых выше.

Определен процесс $\left\{U_{g}(t, \tau)\right\}: U_{g}(t, \tau) u_{\tau}=u(t), U_{g}(t, \tau): H \rightarrow H, t \geqslant \tau$, $\tau \in \mathbb{R}$, где $u(t)$ - решение задачи (4.3)-(4.4).

Для описания пространства символов $\Sigma$ уравнения (4.3) рассмотрим некоторую фиксированную внешнюю силу $g_{0}(s)$, которая является трансляционно компактной функцией в $L_{2}^{\mathrm{loc}}(\mathbb{R} ; H)$. В частности, функция $g_{0}$ ограничена в $L_{2}^{\text {loc }}(\mathbb{R} ; H)$ и $g_{0}$ удовлетворяет $(4.5)$. Пусть $\mathscr{H}\left(g_{0}\right)$ - оболочка $g_{0}$ в $L_{2}^{\text {loc }}(\mathbb{R} ; H)$. Рассмотрим семейство задач Коши

$$
\begin{aligned}
& \partial_{t} u=-\nu L u-B(u, u)+g(t) \equiv A_{g(t)}(u), \\
& \left.u\right|_{t=\tau}=u_{\tau}, \quad g \in \mathscr{H}\left(g_{0}\right) .
\end{aligned}
$$


Очевидно, что для каждой $g \in \mathscr{H}\left(g_{0}\right)$ задача (4.9) имеет единственное решение $u(t)$ и выполнены оценки (4.6), (4.7) и (4.8). Следовательно, задаче (4.9) соответствует семейство процессов $\left\{U_{g}(t, \tau)\right\}, g \in \mathscr{H}\left(g_{0}\right)$, действующих в $H$. Временнь́м символом уравнения (4.9) служит функция $\sigma(s)=g(s)$. Пространством символов $\Sigma$ является оболочка $\mathscr{H}\left(g_{0}\right)$. В силу сделанных предположений, $\Sigma=\mathscr{H}\left(g_{0}\right) \Subset L_{2}^{\text {loc }}(\mathbb{R} ; H)$. Следовательно, пространство символов является компактным метрическим пространством.

Рассмотрим полугруппу $\{\mathscr{S}(t)\}$, действующую в $H \times \mathscr{H}\left(g_{0}\right)$ по формуле $(3.2)$, т. e.

$$
\mathscr{S}(t)\left(u_{0}, g\right)=\left(U_{g}(t, 0) u_{0}, g(t+s)\right), \quad t \geqslant 0 .
$$

В [7] доказано, что семейство процессов $\left\{U_{g}(t, \tau)\right\}, g \in \mathscr{H}\left(g_{0}\right)$, порожденное задачей (4.9), является равномерно (по $\left.g \in \mathscr{H}\left(g_{0}\right)\right)$ ограниченным, равномерно компактным и $\left(H \times \mathscr{H}\left(g_{0}\right), H\right)$-непрерывным. Следовательно, семейство процессов $\left\{U_{g}(t, \tau)\right\}, g \in \mathscr{H}\left(g_{0}\right)$, удовлетворяет всем условиям теоремы 4.1. Поэтому полугруппа $\{\mathscr{S}(t)\}$ (см. (4.10)) имеет компактный (в $\left.H \times \mathscr{H}\left(g_{0}\right)\right)$ глобальный аттрактор А. Кроме того, множество $\Pi_{1} \mathbf{A}=\mathscr{A}_{\mathscr{H}\left(g_{0}\right)}$ является равномерным (по $\left.g \in \mathscr{H}\left(g_{0}\right)\right)$ аттрактором семейства процессов $\left\{U_{g}(t, \tau)\right\}, g \in \mathscr{H}\left(g_{0}\right)$, и $\Pi_{2} \mathbf{A}=\mathscr{H}\left(g_{0}\right)$ (напомним, что $\Pi_{1}(u, g)=u$ и $\left.\Pi_{2}(u, g)=g\right)$. Наконец, из следствия 4.1 получаем следующий результат.

Теорема 4.2. Если функиия $g_{0}$ является трансляиионно компактной $в$ $L_{2}^{\operatorname{loc}}(\mathbb{R} ; H)$, то семейство процессов $\left\{U_{g}(t, \tau)\right\}, g \in \mathscr{H}\left(g_{0}\right)$, порожденное задачей (4.9), имеет равномерный (по $\left.g \in \mathscr{H}\left(g_{0}\right)\right)$ глобальный аттрактор

$$
\mathscr{A}_{\mathscr{H}\left(g_{0}\right)}=\bigcup_{g \in \mathscr{H}\left(g_{0}\right)} \mathscr{K}_{g}(0),
$$

где $\mathscr{K}_{g}$ - ядро прощесса $\left\{U_{g}(t, \tau)\right\}$. Ядро $\mathscr{K}_{g}$ непусто при любой $g \in \mathscr{H}\left(g_{0}\right)$.

ПРИмеР 4.2 (неавтономные системы реакиии-дифбузии). Рассматривается следующая неавтономная параболическая система:

$$
\partial_{t} u=a \Delta u-f(u, t)+g(x, t),\left.\quad u\right|_{\partial \Omega}=0, \quad x \in \Omega \Subset \mathbb{R}^{n} .
$$

Предполагается, что $a=\left\{a_{i j}\right\}_{i=1, \ldots, N}^{j=1, \ldots, N}$ есть вещественная $(N \times N)$-матрица с положительной симметричной частью $\left(a+a^{*}\right) / 2 \geqslant \beta I, \beta>0$. Неизвестной является вектор-функция $u=u(x, t), u=\left(u^{1}, \ldots, u^{N}\right)$. Функции $g=\left(g^{1}, \ldots, g^{N}\right)$ и $f=\left(f^{1}, \ldots, f^{N}\right)$ считаются известными.

Рассмотрим пространства $H:=\left(L_{2}(\Omega)\right)^{N}, H^{1}:=\left(H_{0}^{1}(\Omega)\right)^{N}$ с нормами $|\cdot|$ и $\|$ • $\|$ соответственно. Предполагается, что $g(\cdot, t) \in L_{2}^{\operatorname{loc}}(\mathbb{R} ; H)$ и при этом

$$
|g|_{L_{2}^{\mathrm{b}}}^{2}=\|g\|_{L_{2}^{\mathrm{b}(\mathbb{R} ; H)}}^{2}:=\sup _{h \in \mathbb{R}} \int_{h}^{h+1}|g(t)|^{2} d t<\infty .
$$


Относительно нелинейного члена предполагается, что $f(v, t) \in C\left(\mathbb{R}^{N} \times \mathbb{R} ; \mathbb{R}^{N}\right)$ и выполнены следующие условия:

$$
\begin{aligned}
& \sum_{i=1}^{N} \gamma_{i}\left|v^{i}\right|^{p_{i}}-C_{1} \leqslant \sum_{i=1}^{N} f^{i}(v, t) v^{i}=f(v, s) \cdot v, \quad \gamma_{k}>0 \\
& \sum_{i=1}^{N}\left|f^{i}(v, s)\right|^{p_{i} /\left(p_{i}-1\right)} \leqslant C_{2}\left(\sum_{i=1}^{N}\left|v^{i}\right|^{p_{i}}+1\right) \quad \forall v \in \mathbb{R}^{N}
\end{aligned}
$$

$p_{1} \geqslant \cdots \geqslant p_{N} \geqslant 2$. Пусть также

$$
\left(f\left(v_{1}, t\right)-f\left(v_{2}, t\right), v_{1}-v_{2}\right) \geqslant-D\left|v_{1}-v_{2}\right|^{2} \quad \forall v_{1}, v_{2} \in \mathbb{R}^{N}, \quad \forall t \in \mathbb{R} .
$$

Система (4.12) снабжается начальными условиями

$$
\left.u\right|_{t=\tau}=u_{\tau}, \quad u_{\tau} \in H=\left(L_{2}(\Omega)\right)^{N} .
$$

В [7] доказано, что задача Коши (4.12)-(4.17) имеет при любом $u_{\tau} \in H$ единственное решение $u(t) \in C\left(\mathbb{R}_{\tau} ; H\right) \cap L_{2}^{\text {loc }}\left(\mathbb{R}_{\tau} ; H^{1}\right) \cap L_{\mathbf{p}}^{\text {loc }}\left(\mathbb{R}_{\tau} ; L_{\mathbf{p}}(\Omega)\right)$, где $L_{\mathbf{p}}(\Omega)=L_{p_{1}}(\Omega) \times \cdots \times L_{p_{N}}(\Omega)$. Справедлива следующая оценка:

$$
|u(t)|^{2} \leqslant|u(\tau)|^{2} e^{-\lambda(t-\tau)}+R_{0}^{2},
$$

где $R_{0}^{2}=2 C_{1} \lambda^{-1}+\lambda^{-1}\left(1+\lambda^{-1}\right)|g|_{L_{2}^{\mathrm{b}}}^{2}, \lambda=\beta \lambda_{1}, \lambda_{1}$ - первое собственное значение оператора $-\Delta$ с нулевыми граничными условиями. Кроме того,

$$
\begin{gathered}
|u(t)|^{2}+\beta \int_{\tau}^{t}\|u(s)\|^{2} d s+2 \sum_{i=1}^{N} \gamma_{i} \int_{t}^{t+1}\left\|u^{i}(s)\right\|_{L_{p_{i}}}^{p_{i}} d s \\
\leqslant|u(\tau)|^{2}+2 C_{1}+\lambda^{-1} \int_{\tau}^{t}|g(s)|^{2} d s \\
(t-\tau)\|u(t)\|^{2} \leqslant C_{3}(t-\tau)|u(\tau)|^{2}+C_{4}(t-\tau)
\end{gathered}
$$

где $C_{3}(z)$ и $C_{4}(z)$ - монотонные функции $z=t-\tau$.

Следовательно, имеется процесс $\left\{U_{\sigma}(t, \tau)\right\}$, действующий в пространстве $H$ и отвечающий задаче (4.12)-(4.17): $U_{\sigma}(t, \tau): H \rightarrow H, t \geqslant \tau, \tau \in \mathbb{R}$. Символом системы (4.12) служит пара функций $\sigma(s):=(f(v, s), g(x, s)), s \in \mathbb{R}$.

Пусть задан некоторый начальный символ $\sigma_{0}(s)=\left(f_{0}(v, s), g_{0}(x, s)\right)$. Предполагается, что функция $g_{0}(\cdot, s) \in L_{2}^{\text {loc }}(\mathbb{R} ; H)$ является трансляционно компактной в $L_{2}^{\text {loc }}(\mathbb{R} ; H)$. В частности, $g_{0}$ удовлетворяет $(4.13)$. Оболочка $\mathscr{H}\left(g_{0}\right)$ компактна в $L_{2}^{\text {loc }}(\mathbb{R} ; H)$.

Относительно функции $f_{0}(v, s)$ предполагается, что она удовлетворяет (4.14), (4.15) и (4.16). Дополнительно предполагается, что $f_{0}(\cdot, s)$ - трансляционно компактная функция в $C^{\text {loc }}\left(\mathbb{R} ; \mathscr{M}_{1}\right)$, где пространство $\mathscr{M}_{1}:=C\left(\mathbb{R}^{N} ; \mathbb{R}^{M}\right)$ снабжено следующей локальной топологией равномерной сходимости: по определению, $\psi^{(m)}(\cdot) \rightarrow \psi(\cdot)(m \rightarrow \infty)$ в $\mathscr{M}_{1}$, если для любого $R>0$

$$
\max _{|v| \leqslant R}\left|\psi^{(m)}(v) \rightarrow \psi(v)\right|_{\mathbb{R}^{M}} \rightarrow 0 \quad(m \rightarrow \infty) .
$$


Введенная топология является метризуемой, а соответствующее метрическое пространство $\mathscr{M}_{1}$ - полное (см. [7]). Из условия (4.15) вытекает, что функция $f_{0}(v, s)$ ограничена на любом цилиндре $Q(R)=\{(v, s)|| v \mid \leqslant R, s \in \mathbb{R}\}$. В [7] установлен следующий критерий трансляционной компактности: функция $f_{0}(v, s)$ трансляционно компактна в $C^{\text {loc }}\left(\mathbb{R} ; \mathscr{M}_{1}\right)$ тогда и только тогда, когда при любом $R>0$ функция $f_{0}(v, s)$ ограничена и равномерно непрерывна на $Q(R)$, T. e.

$$
\begin{gathered}
\left|f_{0}\left(v_{1}, s_{1}\right)-f_{0}\left(v_{2}, s_{2}\right)\right|_{\mathbb{R}^{M}} \leqslant \\
\quad \alpha_{1}\left(\left|v_{1}-v_{2}\right|+\left|s_{1}-s_{2}\right|, R\right) \\
\forall\left(v_{1}, s_{1}\right),\left(v_{2}, s_{2}\right) \in Q(R), \\
\alpha_{1}(z, R) \rightarrow 0+\quad(z \rightarrow 0+) .
\end{gathered}
$$

Например, функция $f_{0}(v, s)=\sum_{i=1}^{m} f_{i}(v) \zeta_{i}(s)-$ трансляционно компактна в $C^{\text {loc }}\left(\mathbb{R} ; \mathscr{M}_{1}\right)$, где функции $f_{i}(v)$ удовлетворяют $(4.14)-(4.16)$, а скалярные функции $\zeta_{i}(s)$ являются трансляционно компактными в $C^{\operatorname{loc}}\left(\mathbb{R} ; \mathbb{R}_{+}\right), \zeta_{i}(s) \geqslant \delta>0$ при всех $t \in \mathbb{R}$ и $i=1, \ldots, m$.

Очевидно, что функция $\sigma_{0}(s)=\left(f_{0}(v, s), g_{0}(x, s)\right)$ трансляционно компактна в $\Xi=C^{\text {loc }}\left(\mathbb{R} ; \mathscr{M}_{1}\right) \times L_{2}^{\text {loc }}(\mathbb{R} ; H)$. Рассмотрим пространство символов $\Sigma=\mathscr{H}\left(\sigma_{0}\right)$, где $\mathscr{H}\left(\sigma_{0}\right)$ - оболочка $\sigma_{0}$ в $\Xi$. Как уже отмечалось, $\mathscr{H}\left(\sigma_{0}\right)$ - полное метрическое пространство.

Изучается семейство уравнений

$$
\partial_{t} u=a \Delta u-f(u, t)+g(x, t) \equiv A_{\sigma(t)}(u),\left.\quad u\right|_{t=\tau}=u_{\tau}, \quad \sigma \in \mathscr{H}\left(\sigma_{0}\right),
$$

с символами $\sigma(s)=(f(v, s), g(x, s)) \in \mathscr{H}\left(\sigma_{0}\right)$. Для любого $\sigma(s)=(f(v, s)$, $g(x, s)) \in \mathscr{H}\left(\sigma_{0}\right)$ функция $f(v, s)$ удовлетворяет неравенствам (4.14)-(4.16) с теми же самыми константами, что и исходная функция $f_{0}(v, s)$, и $|g|_{L_{2}^{\mathrm{b}}}^{2} \leqslant\left|g_{0}\right|_{L_{2}^{\mathrm{b}}}^{2}$. Следовательно, задача (4.22) является корректной для любого $\sigma(s) \in \mathscr{H}\left(\sigma_{0}\right)$ и порождает семейство процессов $\left\{U_{\sigma}(t, \tau)\right\}, \sigma \in \mathscr{H}\left(\sigma_{0}\right)=\Sigma$, действующих в $H$. Любое решение $u(t)$ этой задачи удовлетворяет оценкам (4.18), (4.19) и (4.20).

УтвеРЖДЕНИЕ 4.2. Семейство процессов $\left\{U_{\sigma}(t, \tau)\right\}, \sigma \in \Sigma$, является равномерно ограниченным, равномерно компактным и $(H \times \Sigma, H)$-замкнутым.

Доказательство первых двух свойств приведено в [7]. Там же доказано свойство $(H \times \Sigma, H)$-непрерывности данного семейства процессов при более сильных ограничениях на нелинейную функцию $f(v, s)$. Свойство $(H \times \Sigma, H)$-замкнутости доказывается существенно проще и при более слабых ограничениях, поэтому мы его здесь не приводим.

Таким образом, все требования теоремы 4.1 выполнены для семейства процессов $\left\{U_{\sigma}(t, \tau)\right\}, \sigma \in \Sigma$, соответствующего задаче (4.22), и, следовательно, полугруппа $\{\mathscr{S}(t)\}$, отвечающая семейству процессов $\left\{U_{\sigma}(t, \tau)\right\}, \sigma \in \Sigma$, и действующая в расширенном фазовом пространстве $H \times \Sigma$, имеет компактный глобальный аттрактор $\mathbf{A} \Subset H \times \Sigma$. При этом проекция $\Pi_{1} \mathbf{A}=\mathscr{A}_{\Sigma}$ является (компактным в $H$ ) равномерным (по $\sigma \in \Sigma$ ) глобальным аттрактором семейства процессов $\left\{U_{\sigma}(t, \tau)\right\}, \sigma \in \Sigma$, а проекция $\Pi_{2} \mathbf{A}$ совпадает с $\Sigma$. Наконец, доказана следующая теорема. 
Теорема 4.3. Если временно́й символ $\sigma_{0}(s)=\left(f_{0}(v, s), g_{0}(x, s)\right)$ системь (4.22) является трансляиионно компактной функцией в $\Xi=C^{\mathrm{loc}}\left(\mathbb{R} ; \mathscr{M}_{1}\right) \times$ $L_{2}^{\text {loc }}(\mathbb{R} ; H)$, то семейство проиессов $\left\{U_{\sigma}(t, \tau)\right\}, g \in \mathscr{H}\left(\sigma_{0}\right)$, отвечающее $(4.22)$, имеет равномерный (по $\left.\sigma \in \mathscr{H}\left(\sigma_{0}\right)\right)$ глобальный аттрактор

$$
\mathscr{A}_{\mathscr{H}\left(\sigma_{0}\right)}=\bigcup_{\sigma \in \mathscr{H}\left(\sigma_{0}\right)} \mathscr{K}_{\sigma}(0),
$$

где $\mathscr{K}_{\sigma}$ - ядро прочесса $\left\{U_{\sigma}(t, \tau)\right\}$. При всех $\sigma \in \mathscr{H}\left(\sigma_{0}\right)$ ядро $\mathscr{K}_{\sigma}$ непусто.

ПримеР 4.3 (неавтономное волновое уравнение с диссипащией). В ограниченной области $\Omega \Subset \mathbb{R}^{3}$ рассматривается гиперболическое уравнение вида

$$
\partial_{t}^{2} u+\gamma \partial_{t} u=\Delta u-f(u, t)+g(x, t),\left.\quad u\right|_{\partial \Omega}=0, \quad x \in \Omega, \quad t \in \mathbb{R},
$$

с положительным коэффициентом диссипации $\gamma$. Неизвестной является скалярная функция $u=u(x, t)$. Члены $f(u, t)$ и $g(x, t)$ зависят от времени.

Предполагается, что $f(v, s) \in C^{1}(\mathbb{R} \times \mathbb{R} ; \mathbb{R})$ и выполнены следующие неравенства:

$$
\begin{aligned}
& F(v, s) \geqslant-m v^{2}-C_{m}, \quad F(v, s)=\int_{0}^{v} f(w, s) d w, \\
& f(v, s) v-\gamma_{1} F(v, s)+m v^{2} \geqslant-C_{m} \quad \forall(v, s) \in \mathbb{R} \times \mathbb{R},
\end{aligned}
$$

где $m>0, \gamma_{1}>0, m$ достаточно мало. Пусть $0<\rho<2$ и предполагается, что

$$
\begin{aligned}
\left|f_{v}(v, s)\right| & \leqslant C_{0}\left(1+|v|^{\rho}\right), \quad\left|f_{s}(v, s)\right| \leqslant C_{0}\left(1+|v|^{\rho+1}\right), \\
F_{s}(v, s) & \leqslant \delta^{2} F(v, s)+C_{1} \quad \forall(v, s) \in \mathbb{R} \times \mathbb{R},
\end{aligned}
$$

где $\delta$ - достаточно малое число. Из (4.26) следует, что

$$
|f(v, s)| \leqslant C_{0}^{\prime}\left(1+|v|^{\rho+1}\right), \quad|F(v, s)| \leqslant C_{0}^{\prime}\left(1+|v|^{\rho+2}\right) .
$$

Случай $\rho=2$ является критическим. Он тоже допускает рассмотрение, но для этого требуется более сложная техника, и мы его здесь не разбираем (см., например, [1], [23], [24], а также [25]-[27]).

Предполагается также, что $g(\cdot) \in L_{2}^{\text {loc }}\left(\mathbb{R} ; L_{2}(\Omega)\right)$.

Начальные условия ставятся при $t=\tau$ :

$$
\left.u\right|_{t=\tau}=u_{\tau}(x),\left.\quad \partial_{t} u\right|_{t=\tau}=p_{\tau}(x), \quad \tau \in \mathbb{R} .
$$

Следующий результат является классическим: если $u_{\tau} \in H_{0}^{1}(\Omega)$ и $p_{\tau} \in$ $L_{2}(\Omega)$, то задача (4.23)-(4.29) имеет единственное решение $u(t) \in C\left(\mathbb{R}_{\tau} ; H_{0}^{1}(\Omega)\right)$, $\partial_{t} u(t) \in C\left(\mathbb{R}_{\tau} ; L_{2}(\Omega)\right)$, причем $\partial_{t}^{2} u(t) \in L_{2}^{\text {loc }}\left(\mathbb{R}_{\tau} ; H^{-1}(\Omega)\right)$. Доказательство основано на методе галёркинских приближений. Автономный случай подробно изучен в [1]-[3], [12], обобщения на неавтономный случай сделаны в [7].

Для краткости будем обозначать $y(t)=\left(u(t), \partial_{t} u(t)\right)=(u(t), p(t))$ и $y_{\tau}=$ $\left(u_{\tau}, p_{\tau}\right)=y(\tau)$. Через $E$ обозначается пространство вектор-функций $y(x)=$ $(u(x), p(x))$ с конечной энергетической нормой $\|y\|_{E}^{2}=|\nabla u|^{2}+|p|^{2}$ в $E=H_{0}^{1}(\Omega) \times$ $L_{2}(\Omega)$. Тогда $y(t) \in E$ при всех $t \geqslant 0$. 
Задача (4.23)-(4.29) эквивалентна системе

$$
\left\{\begin{array} { l } 
{ \partial _ { t } u = p , } \\
{ \partial _ { t } u = - \gamma p + \Delta u - f ( u , t ) + g ( x , t ) , \quad u | _ { \partial \Omega } = 0 , }
\end{array} \quad \left\{\begin{array}{l}
\left.u\right|_{t=\tau}=u_{\tau}, \\
\left.p\right|_{t=\tau}=p_{\tau},
\end{array}\right.\right.
$$

которую можно переписать в виде

$$
\partial_{t} y=A_{\sigma(t)}(y),\left.\quad y\right|_{t=\tau}=y_{\tau},
$$

где $\sigma(s)=(f(v, s), g(x, s))$ - символ уравнения (4.30). Следовательно, если $y_{\tau} \in E$, то задача 4.30 имеет единственное решение $y(t) \in C_{\mathrm{b}}\left(\mathbb{R}_{\tau} ; E\right)$. Тогда определен процесс $\left\{U_{\sigma}(t, \tau)\right\}$, действующий в $E$ по формуле $U_{\sigma}(t, \tau) y_{\tau}=y(t)$.

Определим пространство символов для (4.30). Пусть задан некоторый фиксированный символ $\sigma_{0}(s)=\left(f_{0}(v, s), g_{0}(x, s)\right)$. Предположим, что $g_{0}(x, s)$ трансляционно компактна в $L_{2}^{\operatorname{loc}}\left(\mathbb{R} ; L_{2}(\Omega)\right)$, а функция $f_{0}(v, s)$ удовлетворяет (4.24)-(4.27), причем функция $\left(f_{0}(v, s), f_{0 t}(v, s)\right)$ является трансляционно компактной в $C^{\operatorname{loc}}\left(\mathbb{R} ; \mathscr{M}_{2}\right)$. Здесь $\mathscr{M}_{2}$ обозначает пространство функций

$$
\mathscr{M}_{2}:=\left\{\left(\psi(v), \psi_{1}(v)\right), v \in \mathbb{R} \mid\left(\psi, \psi_{1}\right) \in C\left(\mathbb{R} ; \mathbb{R}^{2}\right)\right\},
$$

снабженное следующей локальной топологией равномерной сходимости: по определению, $\left(\psi^{(m)}, \psi_{1}^{(m)}\right) \rightarrow\left(\psi, \psi_{1}\right)(m \rightarrow \infty)$ в $\mathscr{M}_{2}$, если для любого $R>0$

$$
\max _{|v| \leqslant R}\left(\left|\psi^{(m)}(v) \rightarrow \psi(v)\right|+\left|\psi_{1}^{(m)}(v) \rightarrow \psi_{1}(v)\right|\right) \rightarrow 0 \quad(m \rightarrow \infty) .
$$

Пространство $\mathscr{M}_{2}$ является метрическим и полным. В [7] получен следующий критерий: функция $\left(f_{0}(v, s), f_{0 t}(v, s)\right)$ трансляционно компактна в $C^{\mathrm{loc}}\left(\mathbb{R} ; \mathscr{M}_{2}\right)$ тогда и только тогда, когда при любом $R>0$ функция $\left(f_{0}(v, s), f_{0 t}(v, s)\right)$ ограничена и равномерно непрерывна на цилиндре $Q(R):=\{(v, s)|| v \mid \leqslant R, s \in \mathbb{R}\}$.

Из приведенных условий следует, что функция $\sigma_{0}(s)=\left(f_{0}(v, s), g_{0}(x, s)\right)$ является трансляционно компактной в $\Xi=C^{\text {loc }}\left(\mathbb{R} ; \mathscr{M}_{2}\right) \times L_{2}^{\text {loc }}(\mathbb{R} ; H)$. Рассмотрим пространство символов $\Sigma=\mathscr{H}\left(\sigma_{0}\right)$, где $\mathscr{H}\left(\sigma_{0}\right)$ - оболочка $\sigma_{0}$ в $\Xi$. Очевидно, что $\Sigma$ - полное метрическое пространство.

Легко видеть, что для любого символа $\sigma(s)=(f(v, s), g(x, s)) \in \mathscr{H}\left(\sigma_{0}\right)$ функция $f(v, s)$ удовлетворяет неравенствам (4.24)-(4.27) с теми же самыми константами.

Задача (4.30) корректно поставлена для каждого $\sigma(s) \in \mathscr{H}\left(\sigma_{0}\right)$ и порождает семейство процессов $\left\{U_{\sigma}(t, \tau)\right\}, \sigma \in \mathscr{H}\left(\sigma_{0}\right)$, в пространстве $E$. Следующее утверждение подробно доказано в [7].

УтвеРЖДЕНИЕ 4.3. Семейство процессов $\left\{U_{\sigma}(t, \tau)\right\}, \sigma \in \mathscr{H}\left(\sigma_{0}\right)$, coответствующее задаче (4.30), является равномерно (по $\left.\sigma \in \mathscr{H}\left(\sigma_{0}\right)\right)$ ограниченным, причем выполнена оченка

$$
\|y(t)\|_{E}^{2} \leqslant C_{4}\left\|y_{\tau}\right\|_{E}^{\rho+2} \exp (-\beta(t-\tau))+C_{5}, \quad \beta>0,
$$

где $y=U_{\sigma}(t, \tau) y_{\tau}, \sigma \in \Sigma$, константы $C_{i}$ не зависят от $y_{\tau} u \sigma$.

СлеДСтвиЕ 4.2. Семейство процессов $\left\{U_{\sigma}(t, \tau)\right\}, \sigma \in \mathscr{H}\left(\sigma_{0}\right)$, отвечающее задаче (4.30), имеет ограниченное (в E) равномерно поглощающее множество. 
УтВеРЖДЕНИЕ 4.4. Семейство процессов $\left\{U_{\sigma}(t, \tau)\right\}, \sigma \in \mathscr{H}\left(\sigma_{0}\right)$, cоответствующее системе (4.30), является равномерно (по $\sigma \in \Sigma$ ) асимптотически компактным и $\left(E \times \mathscr{H}\left(\sigma_{0}\right), E\right)$-замкнутым.

Свойство асимптотической компактности для диссипативного неавтономного волнового уравнения доказано в [7]. Там же установлена $\left(E \times \mathscr{H}\left(\sigma_{0}\right), E\right)$-непрерывность соответствующего семейства процессов при выполнении более сильных ограничений для нелинейной функции $f(v, s)$. Свойство $\left(E \times \mathscr{H}\left(\sigma_{0}\right), E\right)$ замкнутости доказывается достаточно просто, поэтому мы его здесь не приводим.

Стандартным способом строится полугруппа $\{\mathscr{S}(t)\}$ в расширенном фазовом пространстве $E \times \mathscr{H}\left(\sigma_{0}\right)$, которая соответствует семейству $\left\{U_{\sigma}(t, \tau)\right\}$, $\sigma \in \mathscr{H}\left(\sigma_{0}\right)$. Применяется теорема 4.1 и получается следующий основной результат для диссипативного волнового уравнения.

Теорема 4.4. Если $\sigma_{0}(s)=\left(f_{0}(v, s), g_{0}(x, s)\right)$ - трансляиионно компактная функиия в пространстве $\Xi=C^{\mathrm{loc}}\left(\mathbb{R} ; \mathscr{M}_{2}\right) \times L_{2}^{\mathrm{loc}}\left(\mathbb{R} ; L_{2}(\Omega)\right)$, то семейство процессов $\left\{U_{\sigma}(t, \tau)\right\}, g \in \mathscr{H}\left(\sigma_{0}\right)$, отвечающее задаче $(4.30)$, имеет равномерныи $\left(\right.$ по $\left.\sigma \in \mathscr{H}\left(\sigma_{0}\right)\right)$ глобальный аттрактор

$$
\mathscr{A}_{\mathscr{H}\left(\sigma_{0}\right)}=\bigcup_{\sigma \in \mathscr{H}\left(\sigma_{0}\right)} \mathscr{K}_{\sigma}(0),
$$

где $\mathscr{K}_{\sigma}$ - ядро прощесса $\left\{U_{\sigma}(t, \tau)\right\}$. Ядро $\mathscr{K}_{\sigma}$ непусто для каждого $\sigma \in \mathscr{H}\left(\sigma_{0}\right)$.

\section{5. Неавтономные уравнения с трансляционно компактными символами на $\mathbb{R}_{+}$и их равномерные аттракторы}

В этом разделе будут рассмотрены полупроцессы (случай $\mathscr{R}=\mathbb{R}_{+}$), которые порождаются неавтономными уравнениями вида (2.4), временнь́е символы которых заданы на полуоси времени: $\sigma(s), s \geqslant 0$.

Как обычно, задается некоторый исходный символ $\sigma_{0}(s)$, принадлежащий объемлющему хаусдорфову пространству $\Xi_{+}=\{\xi(s), s \geqslant 0\}$, на котором действует непрерывная полугруппа трансляций $\{T(t), t \geqslant 0\}$. Примеры таких пространств $\Xi_{+}$приведены в разделе 2 . Предполагается, что исходный символ $\sigma_{0}(s)$ является трансляционно компактной функцией в $\Xi_{+}$, т. е. его оболочка $\mathscr{H}_{+}\left(\sigma_{0}\right)$ компактна в $\Xi_{+}\left(\right.$см. определение 2.1 при $\left.\mathscr{R}=\mathbb{R}_{+}\right)$. Тогда множество $\Sigma=\mathscr{H}_{+}\left(\sigma_{0}\right)$ удовлетворяет свойству нестрогой инвариантности $(2.7)$ :

$$
T(t) \mathscr{H}_{+}\left(\sigma_{0}\right) \subseteq \mathscr{H}_{+}\left(\sigma_{0}\right) \quad \forall t \in \mathbb{R}_{+} .
$$

Полугруппа трансляций $\{T(t)\}$ действует на компактном метрическом пространстве $\mathscr{H}_{+}\left(\sigma_{0}\right)$. Следовательно, по теореме 2.1 имеется глобальный аттрактор, который, очевидно, совпадает с $\omega\left(\mathscr{H}_{+}\left(\sigma_{0}\right)\right)$, при этом

$$
\begin{aligned}
& \operatorname{dist}_{\Xi_{+}}\left(T(t) \mathscr{H}_{+}\left(\sigma_{0}\right), \omega\left(\mathscr{H}_{+}\left(\sigma_{0}\right)\right)\right) \rightarrow 0 \quad(t \rightarrow \infty), \\
& T(t) \omega\left(\mathscr{H}_{+}\left(\sigma_{0}\right)\right)=\omega\left(\mathscr{H}_{+}\left(\sigma_{0}\right)\right) \quad \forall t \geqslant 0 .
\end{aligned}
$$


ЗАмечАНИЕ 5.1. Отметим, что структура множества $\omega\left(\mathscr{H}_{+}\left(\sigma_{0}\right)\right)$ может быть очень сложной, когда символом $\sigma_{0}$ служит произвольная трансляционно компактная функция. Более простой случай будет рассмотрен в разделе 7 при изучении асимптотически почти периодических функций.

Опишем равномерный аттрактор семейства полупроцессов, когда пространство символов является оболочкой $\mathscr{H}_{+}\left(\sigma_{0}\right)$. Как обычно, предполагается, что для любого символа $\sigma(s) \in \mathscr{H}_{+}\left(\sigma_{0}\right)$ задача Коши $(2.4)-(2.5)$ при любом $\tau \geqslant 0$ имеет единственное решение $u(t) \in E$ при $t \geqslant \tau$ и $u_{\tau} \in E$. Строим соответствующее семейство полупроцессов $\left\{U_{\sigma}(t, \tau)\right\}, \sigma \in \mathscr{H}_{+}\left(\sigma_{0}\right): U_{\sigma}(t, \tau) u_{\tau}=u(t), t \geqslant \tau$, $\tau \geqslant 0, U_{\sigma}(t, \tau): E \rightarrow E$, где $u(t)$ - решение (2.4)-(2.5). Из теоремы 3.1 получаем следующий результат.

Теорема 5.1. Пусть $\sigma_{0}(s)$ - трансляционно компактная функиия в $\Xi_{+}$. Кроме того, пусть семейство полупрочессов $\left\{U_{\sigma}(t, \tau) \mid t \geqslant \tau, \tau \geqslant 0\right\}, \sigma \in$ $\mathscr{H}_{+}\left(\sigma_{0}\right)$, отвечающее задаче $(2.4)-(2.5)$, является равномерно асимптотически компактным и $\left(\right.$ E $\left.\times \mathscr{H}_{+}\left(\sigma_{0}\right), E\right)$-замкнутым. Пусть $\omega\left(\mathscr{H}_{+}\left(\sigma_{0}\right)\right)-$ глобальный аттрактор непрерывной трансляционной полугруппы $\{T(t)\}$, действующей на $\mathscr{H}_{+}\left(\sigma_{0}\right)$. Тогда равномерный аттрактор $\mathscr{A}_{\mathscr{H}_{+}\left(\sigma_{0}\right)}$ семейства $\left\{U_{\sigma}(t, \tau) \mid\right.$ $t \geqslant \tau, \tau \geqslant 0\}, \sigma \in \mathscr{H}_{+}\left(\sigma_{0}\right)$, существует и совпадает с равномерным аттрактором $\mathscr{A}_{\omega\left(\mathscr{H}_{+}\left(\sigma_{0}\right)\right)}$ семейства $\left\{U_{\sigma}(t, \tau) \mid t \geqslant \tau, \tau \geqslant 0\right\}, \sigma \in \omega\left(\mathscr{H}_{+}\left(\sigma_{0}\right)\right)$ :

$$
\mathscr{A}_{\mathscr{H}_{+}\left(\sigma_{0}\right)}=\mathscr{A}_{\omega\left(\mathscr{H}_{+}\left(\sigma_{0}\right)\right)} \text {. }
$$

СлеДСтвиЕ 5.1. Пусть в теореме $5.1 \sigma_{0}(s)=\sigma_{1}(s)+a(s)$, где $\sigma_{1}-$ трансляиионно компактная функиия в $\Xi_{+}$, и пусть $T(t) a \rightarrow 0(t \rightarrow+\infty)$ в $\Xi_{+}$. Тогда

$$
\mathscr{A}_{\mathscr{H}_{+}\left(\sigma_{0}\right)}=\mathscr{A}_{\mathscr{H}_{+}\left(\sigma_{1}\right)}=\mathscr{A}_{\omega\left(\mathscr{H}_{+}\left(\sigma_{1}\right)\right)} .
$$

Рассмотрим коротко следующие примеры уравнений с частными производными.

ПримеР 5.1 (система Навъе-Стокса с внешней силой, трансляиионно компактной на $\left.\mathbb{R}_{+}\right)$. Предположим, что $g_{0}(t)$ - трансляционно компактная функция в $L_{2}^{\text {loc }}\left(\mathbb{R}_{+} ; H\right)$. Рассмотрим семейство задач Коши

$$
\partial_{t} u+\nu L u+B(u, u)=g(t), \quad g \in \mathscr{H}_{+}\left(g_{0}\right),\left.\quad u\right|_{t=\tau}=u_{\tau}, \quad u_{\tau} \in H, \quad \tau \geqslant 0,
$$

и соответствующее семейство полупроцессов $\left\{U_{g}(t, \tau)\right\}, g \in \mathscr{H}_{+}\left(g_{0}\right)$, действующих в $H$. Это семейство является равномерно (по $\left.g \in \mathscr{H}_{+}\left(g_{0}\right)\right)$ компактным и $\left(H \times \mathscr{H}_{+}\left(g_{0}\right), H\right)$-непрерывным. Доказательство аналогично доказательству из примера 4.1 (см. также [7]). В силу теоремы 5.1 равномерный (по $\left.g \in \mathscr{H}_{+}\left(g_{0}\right)\right)$ аттрактор $\mathscr{A}_{\mathscr{H}_{+}\left(g_{0}\right)}$ семейства полупроцессов $\left\{U_{g}(t, \tau)\right\}, g \in \mathscr{H}_{+}\left(g_{0}\right)$, совпадает с равномерным (по $\left.g \in \omega\left(\mathscr{H}_{+}\left(g_{0}\right)\right)\right)$ аттрактором $\mathscr{A}_{\omega\left(\mathscr{H}_{+}\left(g_{0}\right)\right)}$ задачи

$$
\partial_{t} u+\nu L u+B(u, u)=g(t), \quad g \in \omega\left(\mathscr{H}_{+}\left(g_{0}\right)\right),\left.\quad u\right|_{t=\tau}=u_{\tau}, \quad \tau \geqslant 0,
$$

т. e.

$$
\mathscr{A}_{\mathscr{H}_{+}\left(g_{0}\right)}=\mathscr{A}_{\omega\left(\mathscr{H}_{+}\left(g_{0}\right)\right)} \text {. }
$$


ПримеР 5.2 (неавтономная система реакиии-диффузии с трансляиионно компактными членами на полуоси). Предполагается, что в системе (4.12) функция $g(x, s)=g_{0}(x, t)$ является трансляционно компактной в пространстве $L_{2}^{\text {loc }}\left(\mathbb{R}_{+} ;\left(L_{2}(\Omega)\right)^{N}\right)$, функция $f(v, s)=f_{0}(v, s)$ трансляционно компактна в $C^{\text {loc }}\left(\mathbb{R}_{+} ; \mathscr{M}_{1}\right)$ и $f_{0}$ удовлетворяет условиям (4.14)-(4.16). Положим $\sigma_{0}=$ $\left(f_{0}(v, s), g_{0}(x, s)\right)$. Рассмотрим в качестве пространства символов $\Sigma=\mathscr{H}_{+}\left(\sigma_{0}\right)$, где оболочка взята в $C^{\text {loc }}\left(\mathbb{R}_{+} ; \mathscr{M}_{1}\right) \times L_{2}^{\text {loc }}\left(\mathbb{R}_{+} ;\left(L_{2}(\Omega)\right)^{N}\right)$. Задача $(4.22)$ с символами $\sigma=(h, g) \in \mathscr{H}_{+}\left(\sigma_{0}\right)$ порождает семейство полупроцессов $\left\{U_{\sigma}(t, \tau)\right\}, \sigma \in$ $\mathscr{H}_{+}\left(\sigma_{0}\right)$. Это семейство равномерно (по $\left.\sigma \in \mathscr{H}_{+}\left(\sigma_{0}\right)\right)$ компактно и $\left(\left(L_{2}(\Omega)\right)^{N} \times\right.$ $\left.\mathscr{H}_{+}\left(\sigma_{0}\right),\left(L_{2}(\Omega)\right)^{N}\right)$-замкнуто (см. пример 4.2). Значит, применимы результаты этого раздела. Аттрактор $\mathscr{A}_{\mathscr{H}_{+}\left(\sigma_{0}\right)}$ семейства полупроцессов $\left\{U_{\sigma}(t, \tau)\right\}, \sigma \in$ $\mathscr{H}_{+}\left(\sigma_{0}\right)$, совпадает с аттрактором $\mathscr{A}_{\omega}\left(\mathscr{H}_{+}\left(\sigma_{0}\right)\right)$ семейства полупроцессов, порожденного задачей $(4.22)$ с символами из $\omega\left(\mathscr{H}_{+}\left(\sigma_{0}\right)\right)$.

ПримеР 5.3 (неавтономное волновое уравнение с трансляционно компактными членами на $\mathbb{R}_{+}$). Аналогично примеру 4.3 рассматривается волновое уравнение $(4.23)$. Символ $\sigma_{0}(s)=\left(f_{0}(v, s), g_{0}(x, s)\right)$ исходного уравнения $(4.23)$ предполагается трансляционно компактным в пространстве $C^{\text {loc }}\left(\mathbb{R}_{+} ; \mathscr{M}_{2}\right) \times$

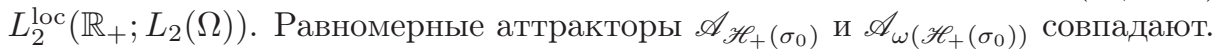

ЗАмечАниЕ 5.2. При сравнении приведенных выше результатов о равномерных глобальных аттракторах семейств процессов и семейств полупроцессов видно, что структура аттракторов для процессов допускает удобное описание в терминах ядер. K сожалению, для общих полупроцессов такое описание не получается. Однако, если сделать некоторые стественные предположения относительно свойств пространства символов, то похожее описание становится возможным. Об этом пойдет речь в следующих разделах.

\section{6. Продолжение полупроцессов до процессов}

В этом разделе будет описана структура равномерного аттрактора семейства полупроцессов в виде объединения сечений ядер процессов, получающихся продолжением исходных процессов на всю ось времени (ср. с теоремой 4.1). Прежде всего заметим, что невозможно дать определение полной траектории полупроцесса, а значит, и ядра полупроцесса, так как тождество (4.1) не имеет смысла при $\tau<0$. Однако возможен иной подход. Пусть задано некоторое семейство полупроцессов $\left\{U_{\sigma}(t, \tau), t \geqslant \tau, \tau \geqslant 0\right\}, \sigma \in \Sigma$, действующих в $E$.

ОПредЕлениЕ 6.1. Семейство процессов $\left\{\widetilde{U}_{\sigma}(t, \tau) \mid t \geqslant \tau, \tau \in \mathbb{R}\right\}, \sigma \in \Sigma_{1}$, где $\Sigma_{1} \subseteq \Sigma$, называется сужением семейства полупроцессов $\left\{U_{\sigma}(t, \tau) \mid t \geqslant \tau\right.$, $\tau \geqslant 0\}, \sigma \in \Sigma$, на $\Sigma_{1}$, если

$$
\widetilde{U}_{\sigma}(t, \tau)=U_{\sigma}(t, \tau) \quad \forall t \geqslant \tau, \quad \tau \geqslant 0, \quad \forall \sigma \in \Sigma_{1} .
$$

Заметим, что сужение относится к пространству символов $\Sigma_{1}\left(\Sigma_{1} \subseteq \Sigma\right)$, в то же время для фиксированного полупроцесса $\left\{U_{\sigma}(t, \tau)\right\}$ с символом $\sigma \in \Sigma_{1}$ можно говорить о его продолжении до процесса $\left\{\widetilde{U}_{\sigma}(t, \tau)\right\}$, имеющего тот же символ $\sigma$. 
ОПРЕДЕЛЕНИЕ 6.2. Будем говорить, что полугруппа $\{T(t)\}$, действующая на $\Sigma, T(t): \Sigma \rightarrow \Sigma, t \geqslant 0$, удовлетворяет свойству обратной единственности, если равенство $T(t) \sigma^{\prime}=T(t) \sigma$ для некоторого $t \geqslant 0$ влечет равенство $\sigma=\sigma^{\prime}$.

ЗАмЕчАниЕ 6.1. В этом разделе рассматриваются только такие пространства символов, для которых полугруппа $\{T(t)\}$ удовлетворяет свойству обратной единственности. Например, это свойство выполнено для неавтономных уравнений с асимптотически почти периодическими символами и для каскадных систем, которые будут рассмотрены далее (см. раздел 8). Вместе с этим, можно построить примеры трансляционно компактных символов, у которых нет этого свойства. Это приводит к более сложному описанию структуры равномерного аттрактора (см. [11]).

Теорема 6.1. Пусть семейство полупроиессов $\left\{U_{\sigma}(t, \tau) \mid t \geqslant \tau \geqslant 0\right\}, \sigma \in \Sigma$, удовлетворяет условиям теоремы 3.1 (при $\left.\mathscr{R}=\mathbb{R}_{+}\right)$, а полугруппа $\{T(t)\}$ удовлетворяет свойству обратной единственности. Обозначим через $\omega(\Sigma)$ глобальный аттрактор $\{T(t)\}$. Тогда существует единственное семейство процессов $\left\{\widetilde{U}_{\sigma}(t, \tau) \mid t \geqslant \tau, \tau \in \mathbb{R}\right\}, \sigma \in \omega(\Sigma)$, которое является сужением семейства полупрочессов $\left\{U_{\sigma}(t, \tau) \mid t \geqslant \tau, \tau \geqslant 0\right\}, \sigma \in \Sigma$, на $\omega(\Sigma)\left(\Sigma_{1}=\omega(\Sigma)\right)$. Семейство прочессов $\left\{\widetilde{U}_{\sigma}(t, \tau)\right\}, \sigma \in \omega(\Sigma)$, удовлетворяет условиям теоремы 4.1 (при $\mathscr{R}=\mathbb{R})$ и, следовательно, имеет равномерный $($ по $\sigma \in \omega(\Sigma))$ аттрактор $\mathscr{A}_{\omega(\Sigma)} \Subset E$. Кроме того,

$$
\mathscr{A}_{\Sigma}=\mathscr{A}_{\omega(\Sigma)}=\bigcup_{\sigma \in \omega(\Sigma)} \mathscr{K}_{\sigma}(0)
$$

где $\mathscr{K}_{\sigma}-$ ядро прочесса $\left\{\widetilde{U}_{\sigma}(t, \tau)\right\}$.

Из теоремы 6.1 следует, что для любого $\sigma \in \omega(\Sigma)$ полупроцесс $\left\{U_{\sigma}(t, \tau) \mid t \geqslant \tau\right.$, $\tau \geqslant 0\}$ имеет единственное продолжение до процесса $\left\{\widetilde{U}_{\sigma}(t, \tau) \mid t \geqslant \tau, \tau \in \mathbb{R}\right\}$.

ДокАЗАтЕльСтво. Зафиксируем произвольный символ $\sigma \in \omega(\Sigma)$ и построим процесс $\left\{\widetilde{U}_{\sigma}(t, \tau) \mid t \geqslant \tau, \tau \in \mathbb{R}\right\}$. Множество $\omega(\Sigma)$ является глобальным аттрактором полугруппы $\{T(t)\}$. Следовательно, в силу строгой инвариантности $\omega(\Sigma)$ найдется такая полная траектория $\gamma(s), s \in \mathbb{R}$, этой полугруппы, что $\gamma(0)=\sigma$. В силу свойства обратной единственности эта траектория единственна, и полугруппа $\{T(t), t \geqslant 0\}$ (на $\omega(\Sigma))$ имеет единственное продолжение для отрицательных $t$ до группы, которую мы тоже обозначим $\{T(t), t \in \mathbb{R}\}$. Поскольку множество $\omega(\Sigma)$ компактно, группа $\{T(t), t \in \mathbb{R}\}$ очевидно непрерывна на $\omega(\Sigma)$. Зададим процесс $\left\{\widetilde{U}_{\sigma}(t, \tau)\right\}$ формулой

$$
\widetilde{U}_{\sigma}(t, \tau)=U_{T(\tau) \sigma}(t-\tau, 0), \quad t \geqslant \tau, \quad \tau \in \mathbb{R}
$$

Проверим, что это семейство отображений является процессом в $E$. В самом деле, при $t=\tau$ получаем $\widetilde{U}_{\sigma}(\tau, \tau)=U_{T(\tau-\tau) \sigma}(\tau-\tau, 0)=U_{\sigma}(0,0)=\mathrm{Id}-$ тождественный оператор при всех $\tau \in \mathbb{R}$. Чтобы проверить мультипликативное 
свойство, используем (6.2) при $t \geqslant s \geqslant \tau, \tau \in \mathbb{R}$ :

$$
\begin{aligned}
\widetilde{U}_{\sigma}(t, s) \widetilde{U}_{\sigma}(s, \tau) & =U_{T(s) \sigma}(t-s, 0) U_{T(\tau) \sigma}(s-\tau, 0) \\
& =U_{T(s-\tau) T(\tau) \sigma}(t-s, 0) U_{T(\tau) \sigma}(s-\tau, 0) \\
& =U_{T(\tau) \sigma}(t-\tau, s-\tau) U_{T(\tau) \sigma}(s-\tau, 0) \\
& =U_{T(\tau) \sigma}(t-\tau, 0)=\widetilde{U}_{\sigma}(t, \tau) .
\end{aligned}
$$

Здесь применялось тождество (3.1) и свойство мультипликативности (1.2) для полупроцессов. Утверждается, что семейство процессов $\left\{\widetilde{U}_{\sigma}(t, \tau)\right\}, \sigma \in \omega(\Sigma)$, является сужением семейства полупроцессов $\left\{U_{\sigma}(t, \tau)\right\}, \sigma \in \Sigma$, на множество $\omega(\Sigma)$. Пусть $\tau \geqslant 0$. Тогда из трансляционного тождества и из (6.2) вытекает что

$$
\widetilde{U}_{\sigma}(t, \tau)=U_{T(\tau) \sigma}(t-\tau, 0)=U_{\sigma}(t, \tau) .
$$

Очевидно, что для любых фиксированных $t$ и $\tau, t \geqslant \tau, \tau \in \mathbb{R}$, отображение

$$
(u, \sigma) \mapsto U_{T(\tau) \sigma}(t-\tau, 0) u
$$

замкнуто из $E \times \omega(\Sigma)$ в $E$. Очевидно также, что семейство процессов $\left\{\widetilde{U}_{\sigma}(t, \tau)\right\}$, $\sigma \in \omega(\Sigma)$, является равномерно (по $\sigma \in \omega(\Sigma)$ ) асимптотически компактным. Покажем, что трансляционное тождество (3.1) выполнено при всех $\tau \in \mathbb{R}$. По формуле (6.2) находим, что

$$
\widetilde{U}_{\sigma}(t+h, \tau+h)=U_{T(\tau+h) \sigma}(t-\tau, 0) .
$$

С другой стороны,

$$
\widetilde{U}_{T(h) \sigma}(t, \tau)=U_{T(\tau) T(h) \sigma}(t-\tau, 0)=U_{T(\tau+h) \sigma}(t-\tau, 0) .
$$

Следовательно,

$$
\widetilde{U}_{\sigma}(t+h, \tau+h)=\widetilde{U}_{T(h) \sigma}(t, \tau) \quad \forall t \geqslant \tau, \quad \tau \in \mathbb{R}, \quad h \geqslant 0 .
$$

Единственность семейства $\left\{\widetilde{U}_{\sigma}(t, \tau)\right\}, \sigma \in \omega(\Sigma)$, следует из свойства обратной единственности $\{T(t)\}$. На самом деле, если для некоторого процесса $\left\{\widehat{U}_{\sigma}(t, \tau)\right\}$, $\sigma \in \omega(\Sigma)$, выполнено равенство $U_{\sigma}(t, \tau)=\widehat{U}_{\sigma}(t, \tau)$ при всех $t \geqslant \tau \geqslant 0$ и любом $\sigma \in \omega(\Sigma)$, то при любом $\tau<0$ и при каждом $t \geqslant \tau$ мы имеем

$$
U_{T(\tau) \sigma}(t-\tau, 0)=\widehat{U}_{T(\tau) \sigma}(t-\tau, 0)=\widehat{U}_{T(-\tau) T(\tau) \sigma}(t-\tau+\tau, \tau)=\widehat{U}_{\sigma}(t, \tau) .
$$

Следовательно,

$$
\widehat{U}_{\sigma}(t, \tau)=\widetilde{U}_{\sigma}(t, \tau) \quad \forall t \geqslant \tau, \quad \tau<0, \quad \forall \sigma \in \omega(\Sigma),
$$

откуда получаем совпадение этих семейств процессов при всех $t \geqslant \tau$ и $\tau \in \mathbb{R}$.

Наконец, осталось проверить (6.1). Заметим, что полугруппа $\{\widetilde{S}(t)\}$, соответствующая семейству процессов $\left\{\widetilde{U}_{\sigma}(t, \tau)\right\}, \sigma \in \omega(\Sigma)$, и действующая в $E \times \omega(\Sigma)$ по формуле $(3.2)$, совпадает на $E \times \omega(\Sigma)$ с полугруппой $\{S(t)\}$, по- 
строенной по семейству полупроцессов $\left\{U_{\sigma}(t, \tau)\right\}, \sigma \in \Sigma$, и заданной той же формулой

$$
\widetilde{S}(t)(u, \sigma)=\left(\widetilde{U}_{\sigma}(t, 0) u, T(t) \sigma\right)=\left(U_{\sigma}(t, 0) u, T(t) \sigma\right)=S(t)(u, \sigma)
$$

для любого $t \geqslant 0$ и всех $(u, \sigma) \in E \times \omega(\Sigma)$. Из теорем 4.1 и 3.1 заключаем, что полугруппы $\{\widetilde{S}(t)\}$ и $\{S(t)\}$ имеют аттракторы $\widetilde{\mathbf{A}}$ и $\mathbf{A}$, которые совпадают: $\widetilde{\mathbf{A}}=\mathbf{A}$. Это тождество влечет равенство 6.1. Теорема доказана.

\section{7. Неавтономные уравнения с асимптотически почти периодическими символами}

Продолжим изучение семейств полупроцессов (случай $\mathscr{R}=\mathbb{R}_{+}$), начатое в разделе 5 , при условии, что исходный временной символ $\sigma_{0}(s), s \geqslant 0$, уравнения (2.4) является трансляционно компактной функцией в $C_{\mathrm{b}}\left(\mathbb{R}_{+} ; \mathscr{M}\right)$ (или в $\left.L_{p}^{\mathrm{b}}\left(\mathbb{R}_{+} ; \mathscr{E}\right)\right)$. Напомним, что метрика в $C_{\mathrm{b}}\left(\mathbb{R}_{+} ; \mathscr{M}\right)$ равна

$$
\mu_{C_{\mathrm{b}}\left(\mathbb{R}_{+} ; \mathscr{M}\right)}\left(\sigma_{1}(\cdot), \sigma_{2}(\cdot)\right):=\sup _{s \geqslant 0} \mu_{\mathscr{M}}\left(\sigma_{1}(s), \sigma_{2}(s)\right),
$$

а норма в $L_{p}^{\mathrm{b}}\left(\mathbb{R}_{+} ; \mathscr{E}\right)$ задается формулой

$$
\left\|\sigma_{0}(s)\right\|_{L_{p}^{\mathrm{b}\left(\mathbb{R}_{+} ; \mathscr{E}\right)}}^{p}:=\sup _{t \geqslant 0} \int_{t}^{t+1}\left\|\sigma_{0}(s)\right\|_{\mathscr{E}}^{p} d s .
$$

Трансляционно компактные функции в $C_{\mathrm{b}}\left(\mathbb{R}_{+} ; \mathscr{M}\right)$ иногда называют асимптотически почти периодическими, а в $L_{p}^{\mathrm{b}}\left(\mathbb{R}_{+} ; \mathscr{E}\right)$ - асимптотически почти периодическими по Степанову.

Рассмотрим оболочку $\mathscr{H}_{+}\left(\sigma_{0}\right)$ исходного символа $\sigma_{0}$ в пространстве $C_{\mathrm{b}}\left(\mathbb{R}_{+} ; \mathscr{M}\right)$ (или в $\left.L_{p}^{\mathrm{b}}\left(\mathbb{R}_{+} ; \mathscr{E}\right)\right)$, которая служит пространством символов уравнения $(2.4)$. Множество $\mathscr{H}_{+}\left(\sigma_{0}\right)$ компактно в $C_{\mathrm{b}}\left(\mathbb{R}_{+} ; \mathscr{M}\right)\left(\right.$ или в $\left.L_{p}^{\mathrm{b}}\left(\mathbb{R}_{+} ; \mathscr{E}\right)\right)$.

Изучается семейство задач с символами $\sigma(s) \in \Sigma=\mathscr{H}_{+}\left(\sigma_{0}\right) \Subset C_{\mathrm{b}}\left(\mathbb{R}_{+} ; \mathscr{M}\right)$ (или $\left.\mathscr{H}_{+}\left(\sigma_{0}\right) \Subset L_{p}^{\mathrm{b}}\left(\mathbb{R}_{+} ; \mathscr{E}\right)\right)$ :

$$
\partial_{t} u=A_{\sigma(t)}(u),\left.\quad u\right|_{t=\tau}=u_{\tau}, \quad u_{\tau} \in E, \quad t \geqslant \tau, \quad \tau \geqslant 0 .
$$

Как и в разделе 5 , предполагаются выполненными условия теоремы 5.1, и, следовательно, семейство полупроцессов $\left\{U_{\sigma}(t, \tau)\right\}, \sigma \in \mathscr{H}_{+}\left(\sigma_{0}\right)$, имеет аттрактор $\mathscr{A}_{\mathscr{H}_{+}\left(\sigma_{0}\right)}$, который совпадает с аттрактором $\mathscr{A}_{\omega\left(\mathscr{H}_{+}\left(\sigma_{0}\right)\right)}$ семейства полупроцессов $\left\{U_{\sigma}(t, \tau)\right\}, \sigma \in \omega\left(\mathscr{H}_{+}\left(\sigma_{0}\right)\right)$, где $\omega\left(\mathscr{H}_{+}\left(\sigma_{0}\right)\right)$ - аттрактор полугруппы трансляций $\{T(t)\}$ на $\mathscr{H}_{+}\left(\sigma_{0}\right)$.

В [7] доказано, что для любой асимптотически почти периодической функции $\sigma_{0}(s) \in C_{\mathrm{b}}\left(\mathbb{R}_{+} ; \mathscr{M}\right)$ существует единственная почти периодическая функция $\sigma_{1}(s) \in C_{\mathrm{b}}(\mathbb{R} ; \mathscr{M})$ такая, что

$$
\mu_{\mathscr{M}}\left(\sigma_{0}(t), \sigma_{1}(t)\right) \rightarrow 0 \quad(t \rightarrow+\infty) .
$$

Соответственно, если $\sigma_{0}(s) \in L_{p}^{\mathrm{b}}\left(\mathbb{R}_{+} ; \mathscr{E}\right)$ - асимптотически почти периодическая функция в смысле Степанова, то

$$
\int_{t}^{t+1}\left\|\sigma_{0}(s)-\sigma_{1}(s)\right\|_{\mathscr{E}}^{p} d s \rightarrow 0 \quad(t \rightarrow+\infty)
$$


для единственной почти периодической в смысле Степанова функции $\sigma_{1}(s) \in$ $L_{p}^{\mathrm{b}}(\mathbb{R} ; \mathscr{E})$. Кроме того,

$$
\mathscr{H}_{+}\left(\sigma_{0}\right)=\left\{T(h) \sigma_{0}, h \geqslant 0\right\} \cup \Pi_{+} \mathscr{H}\left(\sigma_{1}\right),
$$

где $\Pi_{+}$обозначает оператор ограничения на $\mathbb{R}_{+}$.

Заметим, что для любых почти периодических функций $\sigma(s), \sigma^{\prime}(s) \in C_{\mathrm{b}}(\mathbb{R} ; \mathscr{M})$ выполнено равенство

$$
\begin{aligned}
\mu_{C_{\mathrm{b}}(\mathbb{R}, \mathscr{M})}\left(\sigma, \sigma^{\prime}\right) & =\sup _{s \in \mathbb{R}} \mu_{\mathscr{M}}\left(\sigma(s), \sigma^{\prime}(s)\right)=\sup _{s \in \mathbb{R}_{+}} \mu_{\mathscr{M}}\left(\sigma(s), \sigma^{\prime}(s)\right) \\
& =\mu_{C_{\mathrm{b}}\left(\mathbb{R}_{+} ; \mathscr{M}\right)}\left(\Pi_{+} \sigma, \Pi_{+} \sigma^{\prime}\right) .
\end{aligned}
$$

Аналогично,

$$
\left\|\sigma-\sigma^{\prime}\right\|_{L_{p}^{\mathrm{b}}(\mathbb{R} ; \mathscr{E})}=\left\|\Pi_{+} \sigma-\Pi_{+} \sigma^{\prime}\right\|_{L_{p}^{\mathrm{b}}\left(\mathbb{R}_{+} ; \mathscr{E}\right)},
$$

если $\sigma, \sigma^{\prime} \in L_{p}^{\mathrm{b}}(\mathbb{R} ; \mathscr{E})$ - почти периодические функции в смысле Степанова. Следовательно, $\mathscr{H}\left(\sigma_{1}\right)$ изометрично множеству $\Pi_{+} \mathscr{H}\left(\sigma_{1}\right)$. В дальнейшем мы будем для краткости опускать оператор $\Pi_{+}$. Из (7.2) и (7.4) следует, что оболочка $\mathscr{H}\left(\sigma_{1}\right)$ является глобальным аттрактором полугруппы $\{T(t)\}$ на $\mathscr{H}_{+}\left(\sigma_{0}\right)$ : $\omega\left(\mathscr{H}_{+}\left(\sigma_{0}\right)\right)=\mathscr{H}\left(\sigma_{1}\right), T(h) \mathscr{H}\left(\sigma_{1}\right)=\mathscr{H}\left(\sigma_{1}\right), h \geqslant 0$.

Из (7.5) выводится изометричность полугруппы $\{T(t)\}$ на $\omega\left(\mathscr{H}_{+}\left(\sigma_{0}\right)\right)$ :

$$
\mu_{C_{\mathrm{b}}}\left(T(t) \sigma, T(t) \sigma^{\prime}\right)=\mu_{C_{\mathrm{b}}}\left(\sigma, \sigma^{\prime}\right), \quad t \geqslant 0, \quad \forall \sigma, \sigma^{\prime} \in \omega\left(\mathscr{H}_{+}\left(\sigma_{0}\right)\right)=\mathscr{H}\left(\sigma_{1}\right),
$$

откуда легко следует свойство обратной единственности на $\omega\left(\mathscr{H}_{+}\left(\sigma_{0}\right)\right)$. Тогда применима теорема 6.1. Сужение семейства полупроцессов $\left\{U_{\sigma}(t, \tau)\right\}, \sigma \in$ $\mathscr{H}_{+}\left(\sigma_{0}\right)=\Sigma$, на $\mathscr{H}\left(\sigma_{1}\right)$ образует семейство процессов $\left\{\widetilde{U}_{\sigma}(t, \tau)\right\}, \sigma \in \mathscr{H}\left(\sigma_{1}\right)=$ $\omega(\Sigma)$, которое порождается семейством задач Коши на всей числовой оси $\mathbb{R}$

$$
\partial_{t} u=A_{\sigma(t)}(u), \quad \sigma \in \mathscr{H}\left(\sigma_{1}\right),\left.\quad u\right|_{t=\tau}=u_{\tau}, \quad u_{\tau} \in E, \quad t \geqslant \tau, \quad \tau \in \mathbb{R} .
$$

Ввиду (6.1), равномерный (по $\sigma \in \mathscr{H}_{+}\left(\sigma_{0}\right)$ ) аттрактор задачи (7.1) совпадает с равномерным (по $\left.\sigma \in \mathscr{H}\left(\sigma_{1}\right)\right)$ аттрактором задачи (7.6):

$$
\mathscr{A}_{\mathscr{H}_{+}\left(\sigma_{0}\right)}=\mathscr{A}_{\mathscr{H}\left(\sigma_{1}\right)}=\bigcup_{\sigma \in \mathscr{H}\left(\sigma_{1}\right)} \mathscr{K}(0) .
$$

Значит, для изучения равномерного аттрактора задачи (7.1) с асимптотически почти периодическим символом достаточно исследовать задачу (7.6) с почти периодическим символом. В этом заключается основной результат этого раздела.

Выделим три частных случая задачи (7.1).

Стабилизация на постоянном символе автономного уравнения. Пусть $\sigma_{1}(s) \equiv \sigma_{1}$ не зависит от $s$, т. е. в $(7.2) \mu_{\mathscr{M}}\left(\sigma_{0}(t), \sigma_{1}\right) \rightarrow 0(t \rightarrow+\infty)$, $\sigma_{1} \in \mathscr{M}$, или в $(7.3)$

$$
\int_{t}^{t+1}\left\|\sigma_{0}(s)-\sigma_{1}\right\|_{\mathscr{E}}^{p} d s \rightarrow 0 \quad(t \rightarrow+\infty), \quad \sigma_{1} \in \mathscr{E} .
$$


Тогда предельное уравнение (7.6) примет вид

$$
\partial_{t} u=A_{\sigma_{1}}(u),\left.\quad u\right|_{t=0}=u_{0}, \quad u_{0} \in E .
$$

В этом случае $\mathscr{H}\left(\sigma_{1}\right)=\left\{\sigma_{1}\right\}$. Автономное уравнение (7.8) порождает полугруппу $\{\widetilde{U}(t)\} \quad(\widetilde{U}(t, \tau)=\widetilde{U}(t-\tau))$. Значит, равномерный аттрактор семейства полупроцессов $\left\{U_{\sigma}(t, \tau)\right\}, \sigma \in \mathscr{H}_{+}\left(\sigma_{0}\right)$, соответствующего (7.1), совпадает с глобальным аттрактором полугруппы $\{\widetilde{U}(t)\}$. Отметим, что аналогичные уравнения рассматривались в [28].

Стабилизация на периодическом процессе. Пусть в $(7.1)$ символ $\sigma_{0}(t)$ стремится к периодическому символу,

$\mu_{\mathscr{M}}\left(\sigma_{0}(t), \sigma_{1}(t)\right) \rightarrow 0 \quad(t \rightarrow+\infty) \quad$ или $\int_{t}^{t+1}\left\|\sigma_{0}(s)-\sigma_{1}(s)\right\|_{\mathscr{E}}^{p} d s \rightarrow 0 \quad(t \rightarrow+\infty)$,

где $\sigma_{1}(s+p)=\sigma_{1}(s)$ при всех $s \geqslant 0$. Тогда равномерный аттрактор соответствующего семейства полупроцессов сводится к аттрактору периодического процесса.

Стабилизация на квазипериодическом процессе. Аналогичным методом можно исследовать случай, когда в уравнении (7.2) предельный символ $\sigma_{1}(t)=\varphi\left(\alpha_{1} t, \alpha_{2} t, \ldots, \alpha_{k} t\right)$ является квазипериодической функцией.

Теперь мы кратко опишем равномерные аттракторы конкретных уравнений, аналогичных уравнениям из разделов 4 и 5 .

ПримеР 7.1 (система Навъе-Стокса с асимптотически почти периодическим символом). Предположим, что в примере 5.1 внешняя сила $g(t)=g_{0}(t)$ является асимптотически почти периодической функцией из $L_{2}^{\mathrm{b}}\left(\mathbb{R}_{+} ; H\right)$ в смысле Степанова, т. е., в силу (7.2),

$$
g_{0}(t)=g_{1}(t)+a(t)
$$

где $g_{1} \in L_{2}^{\mathrm{b}}(\mathbb{R} ; H), g_{1}$ - почти периодическая функция и

$$
\int_{t}^{t+1}\|a(s)\|_{H}^{2} d s \rightarrow 0 \quad(t \rightarrow+\infty) .
$$

Рассмотрим семейство задач Коши

$$
\partial_{t} u+\nu L u+B(u, u)=g(t), \quad g \in \mathscr{H}_{+}\left(g_{0}\right),\left.\quad u\right|_{t=\tau}=u_{\tau}, \quad u_{\tau} \in H, \quad \tau \geqslant 0,
$$

которое порождает семейство полупроцессов $\left\{U_{g}(t, \tau)\right\}, g \in \mathscr{H}_{+}\left(g_{0}\right)$, действую-

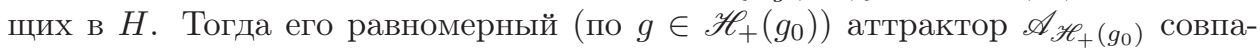
дает с равномерным (по $\left.g \in \mathscr{H}\left(g_{1}\right)\right)$ аттрактором $\mathscr{A}_{\mathscr{H}\left(g_{1}\right)}$ семейства процессов $\left\{U_{g}(t, \tau)\right\}, g \in \mathscr{H}_{+}\left(g_{1}\right)$, задачи $(4.9)$, где вместо $g_{0}$ необходимо подставить $g_{1}$, и из (7.7) находим, что

$$
\mathscr{A}_{\mathscr{H}_{+}\left(g_{0}\right)}=\mathscr{A}_{\mathscr{H}\left(g_{1}\right)}=\bigcup_{g \in \mathscr{H}\left(g_{1}\right)} \mathscr{K}_{g}(0) .
$$


ПримеР 7.2 (системы реакиии-дифбузии с асимптотически почти периодическими членами). Предполагается, что в системе (4.12) члены $f(v, s)=$ $f_{0}(v, s)$ и $g(x, s)=g_{0}(x, t)$ являются асимптотически почти периодическими функциями в пространствах $C_{\mathrm{b}}\left(\mathbb{R}_{+} ; \mathscr{M}_{1}\right)$ и $L_{2}^{\mathrm{b}}\left(\mathbb{R}_{+} ;\left(L_{2}(\Omega)\right)^{N}\right)$ соответственно и, кроме того, $f_{0}$ удовлетворяет условиям (4.14)-(4.16). Пространством символов служит $\Sigma=\mathscr{H}_{+}\left(\sigma_{0}\right) \subseteq \mathscr{H}_{+}\left(f_{0}\right) \times \mathscr{H}_{+}\left(g_{0}\right)$. Задача $(4.22)$ с символом $\sigma=(h, g) \in$ $\mathscr{H}_{+}\left(\sigma_{0}\right)$ порождает семейство полупроцессов $\left\{U_{\sigma}(t, \tau)\right\}, \sigma \in \mathscr{H}_{+}\left(\sigma_{0}\right)$. В соответствии с (7.2) и $(7.3), f_{0}(v, s)=f_{1}(v, s)+k(v, s), g_{0}(x, s)=g_{1}(x, s)+b(x, s)$, где $f_{1}$ и $g_{1}$ - почти периодические функции, $f_{1} \in C_{\mathrm{b}}\left(\mathbb{R}, \mathscr{M}_{1}\right), g_{1} \in L_{2}^{\mathrm{b}}\left(\mathbb{R} ;\left(L_{2}(\Omega)\right)^{N}\right)$ и

$$
\|k(\cdot, t)\|_{\mathscr{M}_{1}}+\int_{t}^{t+1}\|b(s)\|_{\left(L_{2}(\Omega)\right)^{N}}^{2} d s \rightarrow 0 \quad(t \rightarrow+\infty) .
$$

Равномерный аттрактор $\mathscr{A}_{\mathscr{H}_{+}\left(\sigma_{0}\right)}$ семейства полупроцессов $\left\{U_{\sigma}(t, \tau)\right\}, \sigma \in$ $\mathscr{H}_{+}\left(\sigma_{0}\right)$, совпадает с равномерным аттрактором $\mathscr{A}_{\mathscr{H}\left(\sigma_{1}\right)}$ семейства процессов, порожденного задачей (4.22), где вместо $\sigma_{0}$ следует подставить $\sigma_{1}$.

ПримеР 7.3 (волновое уравнение с асимптотически почти периодическими членами). Уравнение (4.23) $\left(f=f_{0}, g=g_{0}\right)$ рассматривается аналогично примерам 7.1 и 7.2. Символом $\sigma_{0}(s)=\left(f_{0}(v, s), g_{0}(x, s)\right)$ уравнения (4.23) служит асимптотически почти периодическая функция

$$
\sigma_{0} \in C_{\mathrm{b}}\left(\mathbb{R}_{+} ; \mathscr{M}_{2}\right) \times L_{2}^{\mathrm{b}}\left(\mathbb{R}_{+} ; L_{2}(\Omega)\right), \quad \sigma_{0}(s)=\sigma_{1}(s)+\xi(s),
$$

где $\xi(s)=(k(v, s), b(x, s))$,

$$
\|k(\cdot, t)\|_{\mathscr{M}_{2}}+\int_{t}^{t+1}\|b(s)\|_{L_{2}(\Omega)}^{2} d s \rightarrow 0 \quad(t \rightarrow+\infty)
$$

и $\sigma_{1}(t)=\left(f_{1}(v, s), g_{1}(x, s)\right)$ - асимптотически почти периодическая функция в пространстве $C_{\mathrm{b}}\left(\mathbb{R} ; \mathscr{M}_{2}\right) \times L_{2}^{\mathrm{b}}\left(\mathbb{R} ; L_{2}(\Omega)\right)$. Равномерные аттракторы $\mathscr{A}_{\mathscr{H}_{+}\left(\sigma_{0}\right)}$ и $\mathscr{A}_{\mathscr{H}\left(\sigma_{1}\right)}$ совпадают.

\section{8. Каскадные системы и их глобальные аттракторы}

Рассмотрим уравнение

$$
\partial_{t} u=A(u, y(t)),\left.\quad u\right|_{t=\tau}=u_{\tau}, \quad u_{\tau} \in E, \quad t \geqslant \tau, \quad \tau \geqslant 0,
$$

в котором $y(t)=T(t) y_{0}, t \geqslant 0$, является траекторией некоторой полугруппы $\{T(t)\}$, действующей в полном метрическом пространстве $\Sigma$. Предполагается, что задача (8.1) с произвольным символом $y(t)=T(t) y_{0}, y_{0} \in \Sigma$, имеет, и притом единственное, решение при всех $\tau \geqslant 0$ и $u_{\tau} \in E$. Следовательно, задача (8.1) порождает семейство полупроцессов $\left\{U_{y_{0}}(t, \tau)\right\}, y_{0} \in \Sigma$, $U_{y_{0}}(t, \tau) u_{\tau}=u(t)$. Символ $\sigma(s)=y(s)$ уравнения (8.1) однозначно определяется своим значением $\left.y\right|_{t=0}=y_{0}$. Очевидно, $y_{0}$ можно отождествить с символом $y(s)$ задачи (8.1), а множество $\Sigma$ можно называть пространством символов. Легко проверить, что трансляционное тождество (3.1) выполнено для семейства полупроцессов $\left\{U_{y_{0}}(t, \tau)\right\}, y_{0} \in \Sigma$, и для полугруппы $\{T(t)\}$. Это позволяет 
построить полугруппу $\{\mathscr{S}(t)\}$ в $E \times \Sigma$ по формуле (3.2). Обычно в приложениях полугруппа $\{T(t)\}$ порождена некоторым автономным дифференциальным уравнением

$$
\partial_{t} y=B(y),\left.\quad y\right|_{t=0}=y_{0},
$$

которое вместе с уравнением (8.1) образует особую ступенчатую систему, которую мы называем каскадной системой.

ЗАмЕчАНИЕ 8.1. Пример 3.4 является частным случаем каскадной системы, в которой полугруппа $\{T(t)\}$, действующая на торе $\mathbb{T}^{k}$, порождена простейшим дифференциальным уравнением

$$
\partial_{t} \omega=\alpha,\left.\quad \omega\right|_{t=0}=\omega_{0} \in \mathbb{T}^{k},
$$

где $\alpha=\left(\alpha_{1}, \ldots, \alpha_{k}\right) \in \mathbb{R}^{k}, \omega=\left(\omega_{1}, \ldots, \omega_{k}\right) \in \mathbb{T}^{k}$. В этом случае полугруппа $\{\mathscr{S}(t)\}$ порождается следующей автономной задачей Коши:

$$
\left\{\begin{array}{l}
\partial_{t} u=A(u, \omega), \quad \partial_{t} \omega=\alpha, \\
\left.u\right|_{t=0}=u_{0},\left.\quad \omega\right|_{t=0}=\omega_{0}, \quad u_{0} \in E, \quad \omega_{0} \in \mathbb{T}^{k},
\end{array}\right.
$$

в которой оператор $A(u, \omega)$ является $2 \pi$-периодическим по каждой переменной $\omega_{j}$.

Вернемся к изучению общей каскадной системы (8.1). Если полугруппа $\{T(t)\}$ замкнута и асимптотически компактна, а семейство полупроцессов $\left\{U_{y_{0}}(t, \tau)\right\}, y_{0} \in \Sigma$, имеет компактное равномерно (по $y_{0} \in \Sigma$ ) притягивающее множество и является $(E \times \Sigma, E)$-замкнутым, то в силу теоремы 3.1 полугруппа $\{\mathscr{S}(t)\}$ имеет компактный глобальный аттрактор А. Его проекция $\Pi_{1} \mathbf{A}=\mathbf{A}_{1}=\mathscr{A}_{\Sigma}$ является равномерным аттрактором семейства полупроцессов $\left\{U_{y_{0}}(t, \tau)\right\}, y_{0} \in \Sigma$, а $\Pi_{2} \mathbf{A}=\mathbf{A}_{2}=\omega(\Sigma)$ - глобальный аттрактор полугруппы $\{T(t)\}$. Кроме того, $\mathscr{A}_{\Sigma}$ совпадает с аттрактором $\mathscr{A}_{\omega(\Sigma)}$ семейства полупроцессов $\left\{U_{y_{0}}(t, \tau)\right\}, y_{0} \in \omega(\Sigma)$. В дополнение к этим результатам, если полугруппа $\{T(t)\}$ удовлетворяет свойству обратной единственности (обычно это свойство выполнено для динамических систем, порождаемых корректными уравнениями), то по теореме 6.1 существует единственное семейство процессов $\left\{U_{y_{0}}(t, \tau), t \geqslant \tau, \tau \in \mathbb{R}\right\}, y_{0} \in \omega(\Sigma)$, которое является сужением семейства полупроцессов $\left\{U_{y_{0}}(t, \tau)\right\}, y_{0} \in \Sigma$, на $\omega(\Sigma)$. Это семейство процессов порождается системой

$$
\begin{aligned}
\partial_{t} u & =A(u, y(t)),\left.\quad u\right|_{t=\tau}=u_{\tau}, \quad u_{\tau} \in E, \\
y(t) & =T(t) y_{0}, \quad t \in \mathbb{R}, \quad y_{0} \in \omega(\Sigma) .
\end{aligned}
$$

Полугруппа $\{T(t)\}$ становится группой на $\omega(\Sigma)$ в силу свойства обратной единственности, и мы находим, что

$$
\mathscr{A}_{\Sigma}=\mathscr{A}_{\omega(\Sigma)}=\bigcup_{y_{0} \in \omega(\Sigma)} \mathscr{K}_{y_{0}}(0)
$$

где $\mathscr{K}_{y_{0}}$ - ядро уравнения (8.3) с символом $y_{0} \in \omega(\Sigma)$. 
В заключение отметим, что построение равномерных аттракторов семейств полупроцессов, порождаемых задачами вида (8.1) с пространством символов $\Sigma$, сводится к задаче (8.3), которая имеет существенно меньшее пространство символов $\omega(\Sigma) \Subset \Sigma$.

В следующих примерах полугруппа $\{T(t)\}$ соответствует некоторому автономному уравнению, а в качестве пространства символов $\Sigma$ выбран шар $B_{R}$ большого радиуса $R$ с центром в начале координат $\Sigma=B_{R}$. Заметим, что в этих примерах глобальные аттракторы $\omega\left(B_{R}\right)$ не зависят от $R$ при $R \gg 1$.

ПримеР 8.1 (смешанная система уравнений реакции-дифбузии и обыкновенных дифберенииальных уравнений). Рассматривается следующая система:

$$
\begin{aligned}
\partial_{t} u & =a \Delta u-f(u, y(t))+g(x, y(t)),\left.\quad u\right|_{\partial \Omega}=0, \quad t \geqslant \tau, \\
\left.u\right|_{t=\tau} & =u_{\tau}, \quad u_{\tau} \in\left(L_{2}(\Omega)\right)^{N}=E, \quad \tau \geqslant 0 ; \\
\frac{d y}{d t} & =-F(y),\left.\quad y\right|_{t=0}=y_{0}, \quad y_{0}, y \in \mathbb{R}^{M},
\end{aligned}
$$

где $x \in \Omega \Subset \mathbb{R}^{n}, a$ - вещественная $(N \times N)$-матрица с положительной симметричной частью $\left(a+a^{*}\right) / 2 \geqslant \beta I, \beta>0, u=u(x, t), u=\left(u^{1}, \ldots, u^{N}\right)$, $g=\left(g^{1}, \ldots, g^{N}\right), f=\left(f^{1}, \ldots, f^{N}\right), y=\left(y^{1}, \ldots, y^{M}\right), F=\left(F^{1}, \ldots, F^{M}\right)$. Предполагается, что $f \in C\left(\mathbb{R}^{N} \times \mathbb{R}^{M} ; \mathbb{R}^{N}\right), g \in C\left([\Omega] \times \mathbb{R}^{M} ; \mathbb{R}^{N}\right), F \in C^{1}\left(\mathbb{R}^{M} ; \mathbb{R}^{M}\right)$. Вектор-функция $f$ удовлетворяет условиям (4.14)-(4.16)), а для функции $F$ выполнены неравенства

$$
(F(y), y) \geqslant m|y|^{2}-C, \quad m>0 .
$$

Легко показать, что при выполнении условий (8.6) задача (8.5) порождает непрерывную полугруппу $\{T(t)\}, T(t): \mathbb{R}^{M} \rightarrow \mathbb{R}^{M}, y(t)=T(t) y_{0}$, имеющую компактное поглощающее множество $\Sigma=B_{R}$, где $R \geqslant R_{0} \gg 1$. Значит, полугруппа $\{T(t)\}$ имеет глобальный аттрактор $\omega\left(B_{R}\right)$. Система $(8.4)$, где $y(t)=$ $T(t) y_{0}$ - решение задачи (8.5), порождает семейство полупроцессов $\left\{U_{y_{0}}(t, \tau)\right\}$, $y_{0} \in B_{R}$, действующих в $E=\left(L_{2}(\Omega)\right)^{N}$. Это семейство является равномерно $\left(\right.$ по $\left.y_{0} \in B_{R}\right)$ компактным и $\left(E \times B_{R}, E\right)$-непрерывным. Следовательно, по теореме 3.1 семейство полупроцессов $\left\{U_{y_{0}}(t, \tau)\right\}, y_{0} \in B_{R}$, имеет равномерный аттрактор $\mathscr{A}_{B_{R}}$. Уравнение (8.5) удовлетворяет свойству обратной единственности, и по теореме 6.1 семейство полупроцессов $\left\{U_{y_{0}}(t, \tau)\right\}, y_{0} \in B_{R}$, имеет единственное сужение на $\omega\left(B_{R}\right)$, а именно семейство процессов $\left\{U_{y_{0}}(t, \tau)\right\}$, $y_{0} \in \omega\left(B_{R}\right)$, с символами $y_{0}$, лежащими на аттракторе $\omega\left(B_{R}\right)$ уравнения (8.5). Множество $\omega\left(B_{R}\right)=\omega\left(B_{R_{0}}\right)$ не зависит от $R$ при $R \geqslant R_{0}$, т. е. $\mathscr{A}_{B_{R}}$ тоже не зависит от $R$ и

$$
\mathscr{A}_{B_{R}}=\mathscr{A}_{\omega\left(B_{R_{0}}\right)}=\bigcup_{y_{0} \in \omega\left(B_{R_{0}}\right)} \mathscr{K}_{y_{0}}(0),
$$

где $\mathscr{K}_{y_{0}}$ - ядро уравнения (8.4) с символом $y_{0} \in \omega\left(B_{R_{0}}\right)$. 
ПримеР 8.2 (уравнение вязкой несжимаемой жидкости с пассивными ингредиентами). Рассматривается система уравнений

$$
\begin{aligned}
\partial_{t} u & =a \Delta u-(y, \nabla) u-f(u, y)+\varphi(x),\left.\quad \frac{\partial u}{\partial \nu}\right|_{\partial \Omega}=0, \\
\left.u\right|_{t=\tau} & =u_{\tau} \\
\partial_{t} y & =-\nu L y-B(y, y)+g(x),\left.\quad y\right|_{\partial \Omega}=0, \\
\left.y\right|_{t=0} & =y_{0}(x) .
\end{aligned}
$$

Здесь $u=\left(u^{1}, \ldots, u^{N}\right)^{\top}, y=\left(y^{1}, y^{2}\right)^{\top}, x \in \Omega \Subset \mathbb{R}^{2}, u_{\tau} \in\left(L_{2}(\Omega)\right)^{N}$ и $y_{0} \in H$. Система (8.8) является автономной 2D-системой Навье-Стокса в пространстве $H$ бездивергентных векторных полей. Неизвестные функции $u^{i}(x, t)$ в $(8.7)$ являются пассивными ингредиентами (концентрации химических веществ, температура и т. п.), не влияющими на скорость $y(x, t)$ течения жидкости. Функция $f(u, y)$ удовлетворяет условиям, подобным (4.14)-(4.16), и $\varphi \in\left(L_{2}(\Omega)\right)^{N}$. Задача 8.8 порождает компактную полугруппу $\{T(t)\}$, действующую в $H$ (см., например, [1]). Пространством символов задачи (8.7) служит множество $\Sigma=$ $B_{R} \subseteq H, R \geqslant R_{0} \gg 1$, где $B_{R}$ - поглощающее множество полугруппы $\{T(t)\}$. Задача (8.7) порождает семейство полупроцессов $\left\{U_{y_{0}}(t, \tau)\right\}, y_{0} \in B_{R}$, действующих в $\left(L_{2}(\Omega)\right)^{N}$. Теоремы 3.1 и 6.1 применимы к этому семейству. Равномерный аттрактор семейства полупроцессов $\left\{U_{y_{0}}(t, \tau)\right\}, y_{0} \in B_{R}$, совпадает с равномерным аттрактором семейства процессов $\left\{U_{y_{0}}(t, \tau)\right\}, y_{0} \in \omega\left(B_{R_{0}}\right)$, где $\omega\left(B_{R_{0}}\right)$ - глобальный аттрактор системы Навье-Стокса (8.8).

\section{Список литературы}

[1] А.В. Бабин, М.И. Вишик, Аттракторы эволющионных уравнений, Наука, М., 1989, 296 с.; англ. пер.: A. V. Babin, M. I. Vishik, Attractors of evolution equations, Stud. Math. Appl., 25, North-Holland, Amsterdam, 1992, x+532 pp.

[2] R. Temam, Infinite-dimensional dynamical systems in mechanics and physics, Appl. Math. Sci., 68, Springer-Verlag, New York, 1988, xvi+500 pp.

[3] J. K. Hale, Asymptotic behavior of dissipative systems, Math. Surveys Monogr., 25, Amer. Math. Soc., Providence, RI, 1988, x+198 pp.

[4] O. Ladyzhenskaya, Attractors for semigroups and evolution equations, Lezioni Lincei, Cambridge Univ. Press, Cambridge, 1991, xii+73 pp.

[5] И. Д. Чуешов, Введение в теорию бесконечномерных диссипативных систем, Университетские лекции по современной математике, Акта, Харьков, 1999, 436 с.; англ. пер.: I. D. Chueshov, Introduction to the theory of infinite-dimensional dissipative systems, University Lectures in Contemporary Mathematics, Acta, Kharkiv, 2002, 418 pp.

[6] A. Haraux, Systèmes dynamiques dissipatifs et applications, Rech. Math. Appl., 17, Masson, Paris, 1991, xii+132 pp.

[7] V.V. Chepyzhov, M.I. Vishik, Attractors for equations of mathematical physics, Amer. Math. Soc. Colloq. Publ., 49, Amer. Math. Soc., Providence, RI, 2002, xii+363 pp.

[8] G.R. Sell, Y. You, Dynamics of evolutionary equations, Appl. Math. Sci., 143, Springer-Verlag, New York, 2002, xiv+670 pp. 
[9] V. V. Chepyzhov, M. I. Vishik, "Non-autonomous dynamical systems and their attractors", Appendix in the book: M. I. Vishik, Asymptotic behaviour of solutions of evolutionary equations, Lezioni Lincee, Cambridge Univ. Press, Cambridge, 1992, 112-150.

[10] V. V. Chepyzhov, M. I. Vishik, "Nonautonomous evolution equations and their attractors", Russian J. Math. Phys., 1:2 (1993), 165-190.

[11] V. V. Chepyzhov, M.I. Vishik, "Attractors of non-autonomous dynamical systems and their dimension", J. Math. Pures Appl. (9), 73:3 (1994), 279-333.

[12] Ж.-Л. Лионс, Некоторые методы решения нелинейных краевых задач, Мир, М., 1972, 587 c.; пер. с фр.: J.-L. Lions, Quelques méthodes de résolution des problèmes aux limites non linéaires, Dunod, Paris; Gauthier-Villars, Paris, 1969, xx+554 pp.

[13] V.V. Chepyzhov, M. I. Vishik, "Attractors of non-autonomous evolution equations with translation-compact symbols", Partial differential operators and mathematical physics (Holzhau, 1994), Oper. Theory Adv. Appl., 78, Birkhäuser, Basel, 1995, 49-60.

[14] V.V. Chepyzhov, M.I. Vishik, "Non-autonomous evolutionary equations with translation-compact symbols and their attractors", C. R. Acad. Sci. Paris Sér. I Math., 321:2 (1995), 153-158.

[15] В. В. Чепыжов, М. И. Вишик, “Аттракторы неавтономных уравнений математической физики с трансляционно-компактными символами", в статье "Совместные заседания семинара им. И.Г. Петровского по дифференциальным уравнениям и математическим проблемам физики и Московского математического общества", УМH, 50:4 (1995), 146-147; англ. пер.: V. V. Chepyzhov, M. I. Vishik, "Attractors of non-autonomous equations of mathematical physics with translation compact symbols", in "Joint sessions of the Petrovskii Seminar on differential equations and mathematical problems of physics and of the Moscow Mathematical Society", Russian Math. Surveys, 50:4 (1995), 802-803.

[16] L. Amerio, G. Prouse, Almost-periodic functions and functional equations, Van Nostrand Reinhold Co., New York-Toronto, ON-Melbourne, 1971, viii+184 pp.

[17] Б. М. Левитан, В. В. Жиков, Почти периодические функиии и дифференииальные уравнения, Изд-во Моск. ун-та, М., 1978, 204 с.

[18] V. V. Chepyzhov, M. Conti, V. Pata, "A minimal approach to the theory of global attractors", Discrete Contin. Dyn. Syst., 32:6 (2012), 2079-2088.

[19] V. Pata, S. Zelik, "Attractors and their regularity for 2-D wave equation with nonlinear damping", Adv. Math. Sci. Appl., 17:1 (2007), 225-237.

[20] О.А. Ладыженская, Математические вопросы динамики вязкой несжимаемой жидкости, Физматгиз, М., 1961, 203 с.; англ. пер.: О. A. Ladyzhenskaya, The mathematical theory of viscous incompressible flow, Gordon and Breach Science Publishers, New York-London, 1963, xiv+184 pp.

[21] Р. Темам, Уравнения Навъе-Стокса. Теория и численный анализ, Мир, М., 1981, 408 с.; пер. с англ.: R. Temam, Navier-Stokes equations. Theory and numerical analysis, revised edition, Stud. Math. Appl., 2, North-Holland, Amsterdam-New York, 1979, x+519 pp.

[22] P. Constantin, C. Foias, Navier-Stokes equations, Chicago Lectures in Math., Univ. Chicago Press, Chicago, IL, 1988, x+190 pp.

[23] О.А. Ладыженская, "О нахождении минимальных глобальных аттракторов для уравнений Навье-Стокса и других уравнений с частными производными”, УМH, 42:6(258) (1987), 25-60; англ. пер.: О. A. Ladyzhenskaya, "On the determination of minimal global attractors for the Navier-Stokes and other partial differential equations", Russian Math. Surveys, 42:6 (1987), 27-73.

[24] J. Arrieta, A. N. Carvalho, J. K. Hale, "A damped hyperbolic equation with critical exponent", Comm. Partial Differential Equations, 17:5-6 (1992), 841-866. 
[25] E. Feireisl, "Attractors for wave equations with nonlinear dissipation and critical exponent", C. R. Acad. Sci. Paris Sér. I Math., 315:5 (1992), 551-555.

[26] M. Grasselli, V. Pata, "On the damped semilinear wave equation with critical exponent", Dynamical systems and differential equations (Wilmington, NC, 2002), Discrete Contin. Dyn. Syst., 2003, suppl., 351-358.

[27] V. Pata, S. Zelik, "A remark on the damped wave equation", Commun. Pure Appl. Anal., 5:3 (2006), 611-616.

[28] С. М. Бородич, "О поведении при $t \rightarrow+\infty$ решений некоторых неавтономных уравнений”, Вестн. Моск. ун-та. Сер. 1. Матем. Мех., 1990, №6, 51-53; англ. пер.: S. M. Borodich, "On behavior as $t \rightarrow+\infty$ of solutions of some nonautonomous equations", Moscow Univ. Math. Bull., 45:6 (1990), 19-21.

В. В. Чепыжов (V. V. Chepyzhov)

Поступила в редакцию

Институт проблем передачи информации

06.02.2013 им. А. А. Харкевича РАН;

Национальный исследовательский университет "Высшая школа экономики"

E-mail: chep@iitp.ru 